\title{
Damping inter-area oscillations using virtual generator based power system stabilizer
}

\author{
Ke Tang and Ganesh K. Venayagamoorthy \\ Real-Time Power and Intelligent Laboratory, Holcombe Department of Electrical and Computer Engineering \\ Clemson University, Clemson, SC 29634, United States
}

\begin{abstract}
In a power system, remote measurements are used by a controller to provide damping torque for inter-area oscillations. In this study, the operating conditions that give rise to inter-area oscillations are investigated using Stochastic Subspace Identification (SSI) based modal analysis. In a power system, generators can be grouped based on coherency and the groups can be represented by Virtual Generators (VGs). Damping inter-area oscillations using Virtual Generator based Power System Stabilizer (VG-PSS) that generates a supplementary control signal to the excitation system of one or more synchronous generators is presented. The generator choice for VG-PSS location is determined based on the generator that has maximum controllability on dominant weakly damped inter-area mode(s) in a power system. Modal analysis using SSI and real-time simulation results on the IEEE 68-bus power system are presented to illustrate the effectiveness of VG-PSSs in damping inter-area oscillations. In addition, the effect of time delays encountered as a result of wide area measurements and communications are considered in the studies presented.
\end{abstract}

Keywords: Damping control, inter-area oscillation, modal analysis, stochastic subspace identification, time-delays, virtual generator

\section{Introduction}

Nowadays due to increased load and limited transmission capability, power systems are constantly pushed towards their stability margin. As a result, oscillations caused by lack of damping torque can be frequently observed. Under different operating conditions, these oscillations may occur in various modes, generally classified as local, intra-area and inter-area modes. Traditional Power System Stabilizers (PSSs) are installed to damp local and intraarea oscillations. Generally, the PSSs use respective generator speed deviation signals as input signals to generate supplementary control signals that are provided to Automatic Voltage Regulators (AVRs). However, locally measured generator speed signal may not contain sufficient information to uncover the characteristics of inter-area 
oscillation modes [1]; as a result, local PSSs are not effective to damp inter-area oscillations. Fortunately, with the deployment of Phasor Measurement Units (PMUs) in the power system, speed signals from remote generators can be made available as additional input signals for the design of advanced damping controllers.

In literature, there exist a lot of papers on supplementary damping control; and a few relevant ones are discussed below [2-11]. An early approach is based on the analysis of power system oscillation modes using Prony Analysis (PA); local PSSs are designed as filters to get rid of specific modes [2]. A real-time implementation of a heuristic approach for online PSS tuning is presented in [3]. A coordinated design of local PSSs and AVRs is presented in [4]. Based on [4], a coordinated PSS design is presented to realize a near-optimal performance for enhanced power system stability in [5]. In [6], low-order power system transfer functions are identified from mathematical models to facilitate robust and practical PSS design. All these designs use local measurements and as a result suffer from insufficient feedback to damp inter-area oscillations.

As for damping controller design using remote measurements, an overview of a Wide Area Control (WAC) system structure is suggested in [7]; the importance of WAC using PMU data to maintain power system stability is elaborated. Though a fuzzy controller making use of PMU measurements of voltages and reactive powers is able to maintain voltage stability, the control approach by capacitor/reactor bank switching may not be swift and accurate enough to damp inter-area oscillations. A two-area system based real-time implementation of a wide-area controller by AVR supplementary control considering communication delays is presented in [8]. The design approach in [8] is completely data-driven, with the control law implemented by a neural network providing supplementary control signals to all generators. For large power systems, it may not be practical to provide supplementary control to all generators. In [9], WAC is applied to a large power system based on a linearized mathematical model; effective measurement and control signals to damp inter-area oscillations are selected using geometric approach. However, the damping control signal is based on a single remote measurement; and an accurate model of a large power system is difficult to obtain. An innovative damping control method is implemented without designing any wide area controllers in [10]. The local PSSs' outputs are combined a matrix gain to generate modulated PSS signals to the AVRs in a 12 bus power system with three generators. The optimal matrix for modulating the initial PSS signals was obtained using the particle swarm optimization algorithm. However, this approach is not directly applicable for larger power systems due to increased matrix size; besides, since the local controllers have been designed only to address the local and intra-area oscillations, they may not work effectively at the frequency range of inter-area 
oscillations. A typical $\mathrm{H}_{\infty}$ wide-area PSS design is proposed in [11]. Despite of the robustness of designed controller to uncertain system parameters, the design with pole-placement constraints may not have a solution; also, since general $\mathrm{H}_{\infty}$ design achieves a controller with the same order with system states; it has to be further reduced to form a PSS at the risk of reduced efficacy. In [12], the concept of Virtual Generator (VG) (elaborated in the following sections) is applied in design of a single-input multiple-output controller using adaptive critic designs; it is indicated that a VG is a mathematical equivalent of a group of generators that tend to oscillate coherently in response to disturbances. However, the damping controller uses only one VG speed; this may not provide sufficient information to damp inter-area oscillations over a wide range of operating conditions.

In this study, power system conditions that give rise to inter-area oscillations using Stochastic Subspace Identification (SSI) based modal analysis are studied; and a new approach to implement supplementary PSS based on VGs (VG-PSS) to damp inter-area oscillations is presented, as shown in Fig. 1. Speed deviations from all the large generation units are remotely measured and sent to a control center, which implements coherency analysis and uses the proposed VG-PSS to generate a supplementary control signal. The generator choice for the supplementary control location is determined based on the generator that has maximum controllability on dominant weakly damped inter-area mode(s) in a power system. The overall flowchart of the suggested design approach is shown in Fig. 2, which is further elaborated in theoretical details in Section 2. The rest of paper is organized as follows: In Section 2, the theory of the proposed method to carry out SSI based modal analysis, the emergence of inter-area oscillations mode(s) in a power system, identification of possible generator excitation system(s) to damp the mode(s), and the development of VG-PSS are described. In Section 3, typical real-time implementation results on the Real-Time Digital Simulator (RTDS) platform for an IEEE 68-bus power system are reported and finally, the conclusion is given in Section 4.

Fig. 1. The diagram of the proposed control scheme with VG-PSS

Fig. 2. The overall flowchart of design approach

\section{Development of VG-PSS}

In this section, a VG-PSS that is heuristically tuned according to modal analysis results, is proposed for supplementary damping control. The modal analysis presented is based on a data-driven system identification 
approach. In addition, the determination of virtual generator(s) based on coherency analysis is presented and the tuning of VG-PSS is elaborated in the following sub-sections.

\subsection{Stochastic Subspace Identification (SSI) Based Modal Analysis}

Besides facilitating controller design, modal analysis also provides a way to exam the efficacy of a controller. Prior to performing modal analysis, it is necessary to obtain a model for the power system without detailed mathematical model of every component that captures the full dynamics. Despite the nonlinearity of power systems, representation of power systems through linear state space has been widely adopted for stability analysis and control based on two facts: first, although individual generators can only be best modelled by nonlinear differential equations, modal analysis based on the linearization of interconnected power system near a nominal operating point can effectively uncover the characteristics of oscillation modes; second, although power systems are subject to changes of operating conditions, these variations are comparatively small comparing to the major systematic topology and generator parameters that remain unchanged; thus, the modal analysis results can be well applied to a range of operating points. Studies presented in references [13-17] include state-space representation of power systems.

In this study, SSI algorithm regards the system matrices as the map from the past data to future data. Generally, in order to design a PSS for stabilizing control, the supplementary signal at the AVR of generators are regarded as the input and the speed deviation responses are taken as the output. Though previous research has used SSI for power system identification [18], it is not applied for Multiple Input Multiple Output (MIMO) case; besides, the matrices $B$ and $C$, were not identified. In the following cases shown, SSI is applied to (a) an input/output based model and (b) an output based model of the power system.

\section{A. Input and Output based Model}

In this case, the power system is modelled as a discrete system with the following expression:

$$
\begin{aligned}
& x(k+1)=A_{1} x(k)+B_{1} u(k) \\
& \omega(k)=C_{1} x(k)+D_{1} u(k)
\end{aligned}
$$

in which the column vectors $x, \omega, u$ are the system states, supplementary inputs and speed deviations respectively. $k$ refers to the time step; in this case $0.064 \mathrm{~s}$ is taken to be the gap between each time step. A Hankel matrix of the past data array $\{u(k) \mid k=0,1, \ldots i+j-2\}$ is defined as, 


$$
U_{p a s t}=U_{0 \mid i-1}=\left[\begin{array}{cccc}
u(0) & u(1) & \ldots & u(j-1) \\
u(1) & u(2) & \ldots & u(j) \\
\ldots & \ldots & \ldots & \ldots \\
u(i-1) & u(i) & \ldots & u(i+j-2)
\end{array}\right]
$$

A Hankel matrix of the future input data array $\{u(k) \mid k=i, i+1, \ldots, 2 i+j-2\}$ is also defined as,

$$
U_{\text {future }}=U_{i \mid 2 i-1}=\left[\begin{array}{cccc}
u(i) & u(i+1) & \ldots & u(i+j-1) \\
u(i+1) & u(i+2) & \ldots & u(i+j) \\
\ldots & \ldots & \ldots & \ldots \\
u(2 i-1) & u(2 i) & \ldots & u(2 i+j-2)
\end{array}\right]
$$

Similarly, the Hankel matrices from the past output data $Y_{\text {past }}$ and future output data $Y_{\text {future }}$ can be defined in the same way as (2) and (3), except for replacing $u$ with $\omega$. Now, a historical data set is defined as,

$$
W_{\text {past }}=\left[U_{\text {past }}^{T}, Y_{\text {past }}^{T}\right]^{T}
$$

It is easy to know from linear system theory that the future output $Y_{\text {future }}$ is impacted not only by historic data set $W_{\text {past }}$, but also by future inputs $U_{\text {future. }}$ Thus, in order to figure out the map from $W_{\text {past }}$ to $Y_{\text {future }}$ with the absence of $U_{\text {future }}$, an oblique projection from $Y_{\text {future }}$ to $W_{\text {past }}$ along $U_{\text {future }}$ is considered and is defined as,

$$
O_{i}=Y_{\text {future }} / U_{\text {future }} W_{\text {past }}
$$

Then singular value decomposition is applied as follows,

$$
\begin{gathered}
O_{i}=U S V^{T}=\left[\begin{array}{ll}
U_{1} & U_{2}
\end{array}\right]\left[\begin{array}{cc}
S_{1} & 0 \\
0 & 0
\end{array}\right]\left[\begin{array}{l}
V_{1}^{T} \\
V_{2}^{T}
\end{array}\right] \triangleq G_{i} X_{i} \\
X_{i}=\operatorname{pinv}\left(G_{i}\right) \cdot O_{i}
\end{gathered}
$$

where $G_{i}=U_{1} S_{1}^{1 / 2}$; "pinv()" stands for the Moore-Penrose pseudo-inverse. It can be seen that each column of $X_{i}$ has the same dimension as the state variable $x$; it actually represents the estimation of system states at each specific time. $X_{i+1}$ can be figured out in a similar way as $X_{i}$ using (4) (7) but replacing $U_{\text {past }}, Y_{\text {past }}, U_{\text {future, }}, Y_{\text {future }}$ with $\bar{U}_{\text {past }}=U_{0 \mid i}$, $\bar{Y}_{\text {past }}=Y_{0 \mid i}, \underline{U}_{\text {future }}=U_{i+1 \mid 2 i-1}, \underline{U}_{\text {future }}=U_{i+1 \mid 2 i-1}$ defined in a similar way as (2) (3). Since the estimation of state variables at every specific moment should satisfy (1), the system matrices $A, B, C, D$ can be estimated from formulae (8) by taking another pseudo-inverse,

$$
\left[\begin{array}{c}
X_{i+1} \\
Y_{i \mid i}
\end{array}\right]=\left[\begin{array}{cc}
A & B \\
C & D
\end{array}\right]\left[\begin{array}{c}
X_{i} \\
U_{i \mid i}
\end{array}\right]
$$


Through this approach, the system matrices can be identified in two steps: (i) injection of PRBS as the system input, and measure the system output; (ii) using formulae $(1) \sim(8)$ to compute system matrices.

\section{B. Output based Model}

There are also some cases when it is not practical to inject PRBS to the power system as supplementary inputs; instead, accurate modal analysis is required to be implemented only according to the measured speed deviations, especially when a contingency happens. Different from the previous case, the power system is equivalent with a discrete model where the input is absent:

$$
\begin{aligned}
& x(k+1)=A_{1} x(k) \\
& \omega(k)=C_{1} x(k)
\end{aligned}
$$

The computation process for $X_{i}$ and $X_{i+1}$ is similar as the previous case except for that there is no need to compute any values related to the input $U_{\text {past }}$ and $U_{\text {future }}$; besides, $W_{\text {past }}$ should be replaced with $Y_{\text {past. }}$ Then, the matrices $A$ and $C$ can be calculated based on (10).

$$
\left[\begin{array}{c}
X_{i+1} \\
Y_{i \mid i}
\end{array}\right]=\left[\begin{array}{c}
A \\
C
\end{array}\right] X_{i}
$$

Through this approach, the system matrices can be identified in two steps: (i) following a contingency in a power system, collect speed response deviations of all generators for a certain window of time after the fault is tripped; (ii) compute system matrices based on a similar set of formulae.

\section{Modal Analysis Based on System Matrices}

Once the system matrices are identified, modal analysis is carried out in order to identify the oscillation modes as well as the controllability factor and observability factor. With the system matrix $A$, use singular value decomposition:

$$
A=V \Lambda W^{T}
$$

where $V W^{T}=I$ and $\Lambda=\operatorname{diag}\left\{\lambda_{1}, \lambda_{2}, \ldots \lambda_{n}\right\}$

Corresponding to the $i^{\text {th }}$ eigenvalue $\lambda_{i}$, the observability factor of the $m^{\text {th }}$ input is calculated as:

$$
\eta_{m i}=c_{m} \cdot v_{i}
$$


where $c_{m}$ is the $m^{\text {th }}$ row of matrix $C$, while $v_{i}$ is the $i^{\text {th }}$ column of matrix $V$. Since both of the two SSI approaches can yield the matrix $C$, the observability factors can be calculated under both cases.

Also, corresponding to $\lambda_{i}$, the controllability factor of the $n^{\text {th }}$ output is calculated as:

$$
\mu_{\text {in }}=w_{i} \cdot b_{n}
$$

where $w_{i}$ is the $i^{\text {th }}$ row of matrix $W$ while $b_{n}$ is the $m^{\text {th }}$ column of matrix $B$. Since only the first SSI approach can yield the matrix $B$, the controllability factors can be calculated only under the first case.

\subsection{Inter-Area Oscillation Analysis}

Inter-area oscillation refers to the phenomenon where two areas in a power system oscillate against each other generally at a low frequency. These areas may contain one or more synchronous generators. When the operating condition is pushed toward stability limit in a power system, oscillations may occur due to lack of damping torque as can be identified using techniques elaborated in Section 2.1 and 2.2. These modes are largely determined by the power system topology, as well as the parameters of generators and transmission lines.

The flow of active power transfer impacts phase-angle stability. Under the same load, increase of active power generation at some generators will cause reduction at others, especially the generator at the slack bus. Accordingly, power flow will change in the power system networks leading to a different operating condition. The location of generations and loads may have a large impact on the oscillations. Generally, oscillation becomes obvious when the load center and generation center do not overlap, especially when some critical transmission lines connecting two areas in the system become heavily loaded. The magnitude of oscillation is impacted by the extent of imbalanced distribution of loads and generations. Occasionally, active power generation in one area of power system may be unanimously increasing, resulting in the increased power flow in some critical transmission lines. Under such condition, it can be observed that the system matrices also varies from the nominal operating condition, the eigenvalues of certain modes may approach to the imaginary axis, causing the rise of oscillation. A serious contingency such as 3-phase fault may trigger power system to lose stability due to increased oscillation, even though the active power flow through the lines are within the transmission limit. Besides, the tripping of crucial transmission lines linking two areas will also result in a weaker power system. Since the bypass of power flows is reduced, the two systems at the sides of transmission lines will be less electromagnetically coherent; and not sufficient damping torque for inter-area oscillation modes may be generated.

\subsection{Structure of VG-PSS}


The traditional structures of local PSSs are composed of a washout component, a lead-lag compensator and a magnitude limiter. Through compensation of phase angle at a specific oscillation mode, a torque is made exactly in opposite phase of speed deviation, leading to increased damping ratio. However, according to the analysis in previous sections, local PSSs may not effectively deal with inter-area oscillation modes, for the generators of maximum controllability are not necessarily with maximum observability. In contrast, a PSS using remote measurement is more capable to provide damping torque for inter-area oscillations. In previous research of Wide Area Control (WAC), the input signal to the controller uses either the speed deviation of one generator of maximum observability or the difference of average speed between two the groups of generators that are oscillating against each other. In this research, on basis of the local PSS structure, a proposed VG- PSS is shown in Fig. 3.

Fig. 3 The proposed structure of VG-PSS.

Taken as the inputs are the virtual generator speeds that are mathematical simplification for groups of coherent generators, as explained in detail in the coming sections. AVR supplementary signal at generator of maximum controllability is taken as the output. This PSS is expected to make use of wide area information to stabilize the power system. Ten parameters are to be decided. The value of $K_{1} \sim K_{\mathrm{M}}$ reflect the impact of each virtual generator speed on the control efficacy; larger values of $K_{i}$ signifies that the $i^{\text {th }}$ virtual generator has larger engagement in this oscillation. $T_{1} \sim T_{4}$ reflect the oscillation frequency as well as the compensation angle. Although the concerned frequency range of the proposed PSS differs from that of local PSSs, its parameters will be configured in an intelligent way as in the analysis of following sections.

In this approach, a heuristic algorithm Particle Swarm Optimization (PSO) is deployed to tune the parameters. The optimal parameters will be reached by repetitively testing the efficacy of each set of WAPSS parameters using 20 particles. During the tuning process, the cost function for each set of parameters is figured out through real-time simulation on Real-Time Digital Simulator (RTDS) with the full model of every component in the power system.

\subsection{Determination of Virtual Generators}

Though damping control is applied using remotely measured signals, the large scale of power system makes it prohibitive to take all generator speed responses as the controller input. Fortunately, previous researches have shown that generators generally oscillate in coherent groups [12]. Generally, generators with small electrical distance show similarity in speed responses following a contingency. A Virtual Generator (VG) is a mathematical equivalent of a group of coherent generators. A complicated multi-machine power system can be significantly simplified with the 
concept of virtual generators. To achieve this, hierarchical clustering algorithm can be applied based on the speed response data of all generators; a dendrogram is yielded so that the generators are classified into several groups under certain threshold.

In this approach, four steps are introduced to obtain the virtual generators in a power system,

i. The power system is disturbed with PRBS injection [19] at the supplementary input site of each generator; and speed responses of all generators are collected.

ii. A hierarchical clustering algorithm is implemented and the diagram is formed showing the coherency of generators.

iii. Through visual inspection, an $\alpha$-cut threshold (as in Fig. 6) is decided and the groupings of generators are formed [18].

iv. The virtual generator speed for each group can be calculated using the formula,

$$
\omega_{V G_{i}}=\frac{H_{i_{1}} \omega_{i_{1}}+H_{i_{2}} \omega_{i_{2}}+\ldots+H_{i_{n}} \omega_{i_{n}}}{H_{i_{1}}+H_{i_{2}}+\ldots+H_{i_{n}}}
$$

It can be seen from (14) that two conditions must be satisfied in order to calculate the VG speeds: first, all the local generator speeds need to be observable by a control center (Fig. 1) that executes the calculation. With the advent of Phasor Measurement Units (PMUs), the real-time variation of frequency at each generator bus can be measured, which is directly linked with the generator speed deviations. For a larger system that covers large geographic domain with large number of generators, PMUs are installed on a limited selection of generators that best represents system dynamics and helps deriving the virtual generator model in each coherent group. Second, the measured frequencies from all generator buses are real-time data flows and thus need to be synchronized. Fortunately, PMU is able to give each measured data with a time stamp with the aid of a Global Positioning System (GPS) radio clock, as a result, the synchronization of real-time measurement at all the generators can be realized. A time-delay is resulted during the synchronization and communication of remote PMU data to the control center. The effect of this time-delay is presented in Section 2.7.

It is computationally and practically challenging to design a supplementary controller making use of all generator speeds especially for a large power system nor is it necessary. A VG is a good representation of a group of generators that behave in a similar manner. Although the concept of VG is originally suggested as mathematical simplification of multiple generators, the determination of VGs is data-based. The clustering/grouping approach uses 
the measured speed responses of generators following PRBS disturbances at the AVRs of all generators, which represents the coherency of generators in a general sense, and is a better approach rather than a clustering approach following a fault or an increase of generation, as has been observed in [20] that the site and type of fault can largely impact the coherency of generators. Considering that the generator coherency is subjected to change of operating conditions and contingencies, variation in generator coherency occurs. However, based on the fact that geologically adjacent generators tend to oscillate coherently, and that contingencies only lead to very slight change of the overall power system topology, the proposed virtual generator modelling based on fixed coherent groups is assumed in this study. Though efficacy of this design is demonstrated in the simulation results, future research will use artificial immune system based adaptive controller to handle the variation of generator coherency, leading to improved damping effectiveness.

\subsection{PSO based VG-PSS Tuning}

Particle Swarm Optimization (PSO) is an adaptive algorithm that heuristically searches best solutions with swarm intelligence; it is widely used for parameter tuning [21]. The parameters to be determined are mirrored to the positions of particles; and a fitness function to be minimized is defined as a satisfactoriness measurement for a set of parameters. To search for a minimal value of fitness function, each particle keeps updating its position by actively moving toward the local and global optimum point, respectively defined as the historical position of its own and of all particles that correspond to the smallest fitness function value. During the optimization, the fitness function is monotonically decreasing until all particles coherently converge to a global optimal position in a final stage. Due to its robustness to nonlinear nonconvex problems, PSO is adopted in this study to optimize the parameters of VGPSS. The following part of this section elaborates technical details for implementation, including the determination of fitness function and the algorithm of parameter updating.

In this study, the position of a particle $p_{i}$ represents the set of all parameters corresponding to Fig. 3, thus $p_{i}=\left[K_{i, 1}, K_{i, 2}, \ldots, K_{i, M}, T_{i, 1}, T_{i, 2}, T_{i, 3}, T_{i, 4}\right]$. Under this set of parameters, speed responses following a fault are obtained through simulation; and a fitness function is required to analyse the speed responses. There are generally two ways to determine the fitness function: through either frequency domain or time domain. Since it is expected that a decreased oscillation be observed from the speed responses with the effect of controller, the damping ratio under the concerned frequency can be taken as the cost function, which is obtained by modal analysis of speed deviation simulation results. Experiments using this frequency-domain based cost function shows ability to reject unexpected 
oscillation modes brought by the new set of parameters obtained in the optimizing process. Alternatively, a cost function can be straightforwardly defined by analysing the time series of speed response curves; for example, the $\mathrm{H}_{2}$-norm (i.e. squared mean average through time) of speed responses can be taking as the cost function. Timedomain based cost function leads to a better final-stage performance despite of slow convergence. It is desired to combine the merits of both these two options: a frequency-domain part of the cost function is expected to play a more important role at the first stage so that the particles approaches an appropriate solution with a fast speed, while a time-domain part of cost function improves the efficacy of controller at the final stage. Thus, the following cost function is adopted:

$$
J=-\alpha \min \left\{\xi_{f}, \xi_{0}\right\}+\sum_{i=1}^{N_{G}} \sum_{j=1}^{N}\left(\Delta \omega_{i}(j)\right)^{2}
$$

in which $\xi_{f}$ denotes the damping ratio near the frequency $f ; \Delta \omega_{i}(j)$ denotes the speed deviation the $i$ 'th generator at the time step $j ; \alpha$ is a large real value; and $\xi_{0}$ denotes a threshold of damping ratio; $N_{G}$ is the generator number, while $N$ is the length of data points. At the beginning of tuning process, since the damping ratio under parameters of most particles may be very low, the judgement of performance is mainly decided first term in (15). However, as the performance of each particle improves so that the damping ratio $\xi_{f}$ is larger than the threshold $\xi_{0}$, the performance is only dependent on the second term that reflects the speed deviation profiles of all generators. The parameters are further adjusted to minimize the second term so that performance is further improved.

Define $p_{i, \text { lbest }}$ and $p_{\text {gbest }}$ as the local and global optimum point of particle $p_{i}$ respectively. At each iteration, all particles are updated through (16) (17). For all $i$ 's.

$$
\begin{gathered}
\Delta v_{i, k}=\eta_{0} v_{i, k-1}+\eta_{1}\left(p_{i, l b e s t}-p_{i, k-1}\right)+\eta_{2}\left(p_{g b e s t}-p_{i, k-1}\right) \\
p_{i, k+1}=p_{i, k}+\Delta v_{i, k}
\end{gathered}
$$

Meanwhile, whenever the cost fitness function is calculated with the updated particle position, $p_{i, l b e s t}$ and $p_{g b e s t}$ are also updated through (18) and (19).

$$
\begin{gathered}
p_{i, \text { lbest }}^{\text {new }}=\min \left\{p_{i, \text { lbest }}^{\text {old }}, p_{i}\right\} ; \\
p_{g}^{\text {new }}=\min \left\{p_{g}^{\text {old }}, p_{i}\right\}
\end{gathered}
$$


In the final stage, all the particles ultimately converge to a solution, and is taken as the solutions. Shown in Fig. 4 is flowchart of PSO parameter-tuning algorithm based on the speed deviation data following PRBS injection at all the AVRs.

Fig. 4 PSO flowchart for VG-PSS parameters tuning algorithm.

\subsection{PSO versus other Design Techniques}

Numerous techniques exist for the design of supplementary damping controllers. Traditional pole-placement is effectively applied for design of single-input single-output controller [22], yet it is not the only way; other approaches such as bacterial foraging algorithm (BFA) and adaptive critic designs (ACDs) have also been used for wide area controller designs, as shown in [23] and [24]. For this study, since the proposed VG-PSS uses speed deviations of multiple VGs, and the contribution of each VG for the control signal is unknown, it is preferred to use the PSO-based heuristic approach in this study. Also, despite its robustness, $\mathrm{H}_{\infty}$-based approach yields several highorder separate controllers linking each VG speed deviation input to the controller output; in contrast, there are limited amount of parameters in the proposed VG-PSS that can be realized with a traditional lead-lag structure. With a simple mathematical form, the VG-PSS establishes a standard schematic for the supplementary damping control, with the parameters best tuned by PSO. The effectiveness of VG-PSS controller is presented in Section 3.

\subsection{Time Delay Compensation}

In the proposed VG-PSS, time delay is incurred about in two processes: (i) $\tau_{\mathrm{PMU}}$ : the communication between the control center and the PMUs; (ii) $\tau_{\text {control }}$ t the communication between the control center and the generator that the supplementary control is applied too. In this study, these two time delays are merged as $\tau=\tau_{\mathrm{PMU}}+\tau_{\text {control }}$, and is compensator inside the VG-PSS by the transfer function given in (20).

$$
G_{C}(s)=K_{C}\left(\frac{1+T_{C 1} s}{1+T_{C 2} s}\right)^{2}
$$

where $T_{C 1}=\frac{1}{\omega} \sqrt{\frac{1+\sin (\omega \tau / 2)}{1-\sin (\omega \tau / 2)}}$ and $T_{C 2}=\frac{1}{\omega} \sqrt{\frac{1-\sin (\omega \tau / 2)}{1+\sin (\omega \tau / 2)}} \cdot \omega$ is the oscillation frequency of concern. $K_{C}$ is normalized so that $\left|G_{C}(j \omega)\right|=1$. This compensator is able to effectively provide a phase angle compensation at the oscillation frequency of concern $\omega$ [25]. For a wide area control system, time delay $\tau$ can be determined with the aid of GPS clock labels included in the PMU data. In this study, $\tau$ is assumed to be time-invariant.

\section{Simulation results}




\subsection{Introduction of IEEE 68-Bus Power System}

This study is based on an IEEE 68-Bus 16-Machine system (also referred to as the NE-NY system) as shown in Fig. 5, which is roughly composed of three subsystems: New England (NE), New York (NY) and Areas 3 through 5. Since G13 serves as the slack bus, and G14-G15 are merely equivalence of three minor areas as three generators, local PSSs are installed on G1-G12 tuned with $\mathrm{H}_{\infty}$ design approach elaborated in [26]. NE and NY are connected by three crucial transmission lines Bus 8-Bus 9, Bus 2-Bus 1, Bus 27-Bus 1, thus inter-area oscillation between NE and NY can be observed.

Fig. 5 IEEE 68-Bus 16-machine system.

For this power system, the hierarchical clustering result is shown in Fig. 6, in which five coherent groups are determined according to a certain alpha-cut, corresponding to five virtual generators.

Fig. 6 The dendrogram of clustering result for IEEE 68-Bus system.

A base operating condition is defined as given in Table A.I. Under this condition, the total power transfer from NE to NY (sum of power flow on lines 8-9, lines 2-1 and line 27-1) is 560 MW. Increase in NE-NY power transfer, $\Delta P$, is investigated for the emergence of inter-area oscillation(s). In case of an increased power transfer, an increase of active power generation in amount of $\Delta P$ is equally shared by generators G1 G9 in NE system, while the same amount of reduction is equally shared by rest of the generators G10 G16 in NE-NY system. Shown in Fig. 7 is the plot of speed deviation responses of G15 under different values of $\Delta P$ post a six-cycle three-phase fault at Bus 31 . Oscillation is prominent when $\Delta P$ reaches $600 \mathrm{MW}$; it further increases as the value of $\Delta P$ rises. By visual inspection of the curves in Fig. 7, it can be seen that the dominant oscillation is around $0.22 \mathrm{~Hz}$, which is verified by the plot of identified modes at $\Delta P=680 \mathrm{MW}$ in shown in Fig. 8.

Fig. 7 Speed deviations plots of G15 for different values of $\Delta P$ (increased active power transfer from NE system to NY system) post-fault at Bus 31.

Fig. 8 Identified system modes for $\Delta P=680 \mathrm{MW}$.

Shown in Fig. 8 is the phasor plot of observability factor of each generator at the mode of $0.22 \mathrm{~Hz}$ when $\Delta P=$ 680MW, which is obtained by analysis of speed deviations after Pseudo Random Binary Signals (PRBSs) are injected through AVRs of all generators. It can be seen that G1 G9 are oscillating against G10 G16; this oscillation is especially prominent on $\mathrm{G} 14, \mathrm{G} 15, \mathrm{G} 16$, which can also be visualized through time-domain curves of speed 
responses. Correspondingly, the controllability factors are shown in Fig. 9. It can be seen that G8 and G9 are the two generators with highest controllability factors.

Fig. 9 The phasor plot of observability factors of G1 G16 obtained through PRBS injection.

Fig. 10 The controllability factors of G1 G16 obtained through PRBS injection.

It is noticed that the generators with highest observability factors are different from those with highest controllability, which is very common in many cases of inter-area oscillations. In another words, the speed deviation of the generator most effective to implement damping control may not be the one that most efficiently reflects the concerned oscillation mode, leading to the conclusion that local damping control is not sufficient for inter-area oscillations.

Following the same procedure, similar plots as Figs. 8 and 9 can be obtained for cases $\Delta P=600 \mathrm{MW}, 630 \mathrm{MW}$, $650 \mathrm{MW}$, which shows the same groups of generators oscillating against each other. G8 and G9 are computed to be generators with highest controllability factors under all cases. Yet as the value of $\Delta P$ changes, the damping ratio changes significantly as listed in Table I.

Table I Oscillation frequencies and corresponding damping ratios for multiple operating conditions

The speed deviation responses in each of the five coherent groups that are equivalent to the five VGs are indicated in Fig. 10 following a 50ms three phase fault at Bus 27 in the standard case in the appendix. The similarity index between each generator and its nearest virtual generator is shown in Table II, showing that the generator speed responses ensemble their corresponding group centers.

Fig. 11 Speed responses of generators in the coherent groups.

Table II The similarity index between each generator and its nearest virtual generator

\subsection{IEEE 68-Bus Power System with VG-PSS}

From the analysis in previous context, the generators with maximum controllability are G8 and G9. The contingency used for PSO tuning is a 50ms fault on Bus 27. Corresponding to different contingencies, three case studies are shown to indicate the efficacy of VG-PSS damping controller. For each of them, the VG-PSS is installed either on G8 or on G9. With VG-PSS on G8 or G9; and the optimized parameters are shown in Table III. In case studies I to III, a time delay of $100 \mathrm{~ms}$ is considered.

Table III The optimized parameters of the VG-PSS 
Case Study I: The system is originally at operating condition II presented in the appendix Table A.1. A 50ms three-phase fault takes place at Bus 2, causing the tripping of transmission lines between Bus 1 and Bus 2. Shown in Fig. 12 are the speed deviation responses of selected generators, the VG-PSS is installed on G9.

Fig. 12 Case Study I -- The speed deviations plots of selected generators with VG-PSS on G9.

Through modal analysis, with no PSSs, the damping ratio is $0.4 \%$, and the power system is marginally stable; with only the local PSSs, the damping ratio is $6.1 \%$, while with only the VG-PSS installation on G9, the damping ratio is $11.6 \%$, with the cooperation of VG-PSS with local PSSs, the damping ratio improved to $28.9 \%$.

The VG-PSS installed on G8 shows similar effectiveness as shown in Fig. 13. In order to verify that the two VG-PSSs can be working cooperatively, Fig. 13 compares the control effect with both VG-PSSs and with only one VG-PSS installed on G8 or G9. It can be concluded that, when VG-PSSs are installed on both G8 and G9, better damping effectiveness is achieved than VG-PSS installed on each individual generator. Even without installation of local PSSs, the cooperating VG-PSSs are able to provide significant damping torque.

Fig. 13 Case Study I -- The speed deviations plots of selected generators for different sites of VG-PSS installations.

Case Study II.A: With the transmission line Bus1-Bus2 already tripped, the system changes to operating condition III.A in the appendix Table A.1. A 50ms three-phase fault happened at Bus 8. Shown in Fig. 14 are the speed deviation responses of selected generators.

Fig. 14 Case Study II.A -- The speed deviations plots of selected generators with VG-PSS on G9.

Without local PSSs and VG-PSS, the power system becomes unstable following the contingency. Through modal analysis, with only the local PSSs, the damping ratio is only $0.5 \%$ which is close to the stability margin; with only the VG-PSS on G9, the damping ratio improves to 7.5\%; and with both local PSSs and the VG-PSS on G9, the damping ratio improves to $16.87 \%$. Fig. 15 compares the control effect with both VG-PSSs and with only one VGPSS installed on G8 or G9 showing the ability of cooperating VG-PSSs to improve the damping effectiveness.

Fig. 15 Case Study II.A -- The speed deviations plots of selected generators for different sites of VG-PSS installations.

Case Study II.B: The system changes to operating condition III.B listed in the appendix Table A.1. Comparing to Case Study II.A, the load on Bus 1 has increased by $100 \%$, from 256 MVA to 512 MVA. The increased active power is supplied by G13. The same contingency as in Case Study II.A is applied and the speed deviation responses of selected generators are shown in Fig. 16. 
Fig. 16 Case Study II.B -- The speed deviations plots of selected generators with VG-PSS on G9 under a new load condition

Without local PSSs and VG-PSS, the power system becomes unstable following the contingency. Through modal analysis, with only the local PSSs, the damping ratio is only $1.5 \%$ which is close to the stability margin; with only the VG-PSS on G9, the damping ratio improves to 7.9\%; and with both local PSSs and the VG-PSS on G9, the damping ratio improves to $17.54 \%$.

Case Study III: The system operation is changed to operating condition IV as shown in the appendix Table A.1. A 50ms three-phase fault is applied at Bus 27. Shown in Fig. 16 are the speed deviation responses of selected generators.

Fig. 17 Case Study III -- The speed deviations plots of selected generators with VG-PSS on G9.

In this case, whether local PSSs are installed or not, if VG-PSS is not installed, the system becomes unstable following the contingency. With the VG-PSS installed, damping ratio is $6.2 \%$ without local PSSs, and is $11.2 \%$ when local PSSs are installed. Fig. 17 compares the control effect with both VG-PSSs and with only one VG-PSS installed on G8 or G9, and same conclusions can be drawn as for Case Studies I and II.

Fig. 18 Case Study III -- The speed deviations plots of selected generators for different sites of VG-PSS installations.

It can be seen from Figs. $12 \sim 17$ that with the VG- PSS implementation, the power system settles to steady state much faster. Also, the damping ratios are improved. Thus, the designed VG-PSS(s) is able to stabilize the power system for all these three cases. The settling time of the generator speed deviations (shown in Figs. 12, 14 and 16) for case studies I, II and III are given in Table IV. Herein, the settling time is defined as the time it takes for the oscillations to settle within $\pm 5 \%$ of the maximum speed deviation. Again, it can be observed that VG-PSSs in combination with local PSSs provides the minimal settling time in all three case studies.

Table IV Settling time of generator speed deviations for the three case studies

The effectiveness of time-delay compensators is shown in Fig. 18. The comparison of speed responses with and without the time-delay compensators for G9 are plotted for different time delays under Case Study II. It can be seen that without compensation, the control effect worsens as the time-delay increases (Fig. 18c). However, the proposed compensator is able to counteract the time delay and improve the damping effectiveness.

Fig. 19 Impact of time delay for the speed responses at G9. 


\section{$4 \quad$ Conclusion}

In this study, SSI-based modal analysis is used first to identify the operating conditions that give rise to interarea oscillation. It is shown that oscillation occurs when the center of generation does not overlap with the load center. The concept of virtual generators is used to cluster the synchronous generators into several coherent groups in an offline manner. Based on the VGs determined, supplementary wide area controller using virtual generator speeds is developed. The particle swarm optimization algorithm is applied to determine the optimized parameters of VG-PSS. Real-time simulation of the IEEE 68-bus system with VG-PSS(s) have illustrated the enhanced damping of the proposed approach for damping inter-area oscillations and maintaining the stability of a power system.

Future work involves the application of online-clustering based adaptive wide area damping controller. For larger power systems, multiple inter-area modes may exist; therefore several VG-PSSs may be necessary to damp all oscillations. 


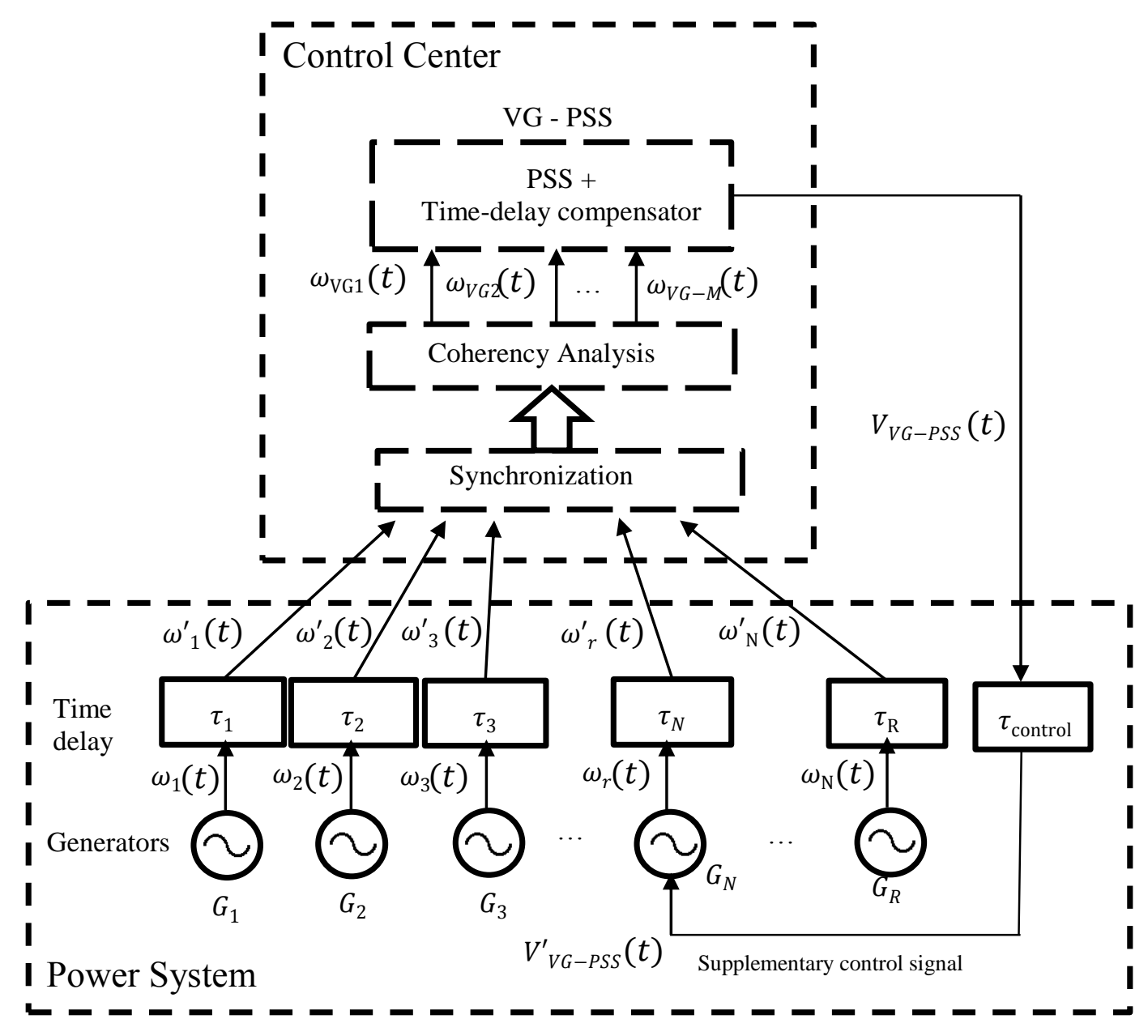

Fig. 1. The diagram of the proposed control scheme with VG-PSS

$\left(\omega^{\prime}{ }_{i}(t)=\omega_{i}\left(t+\tau_{i}\right)\right)$ 


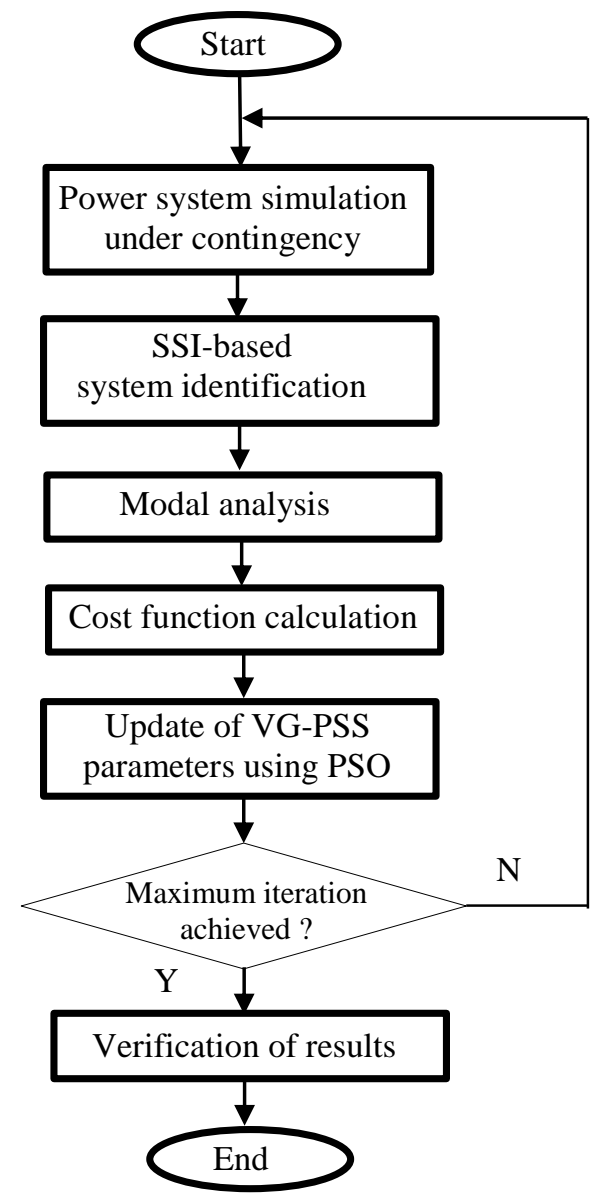

Fig. 2. The overall flowchart of design approach

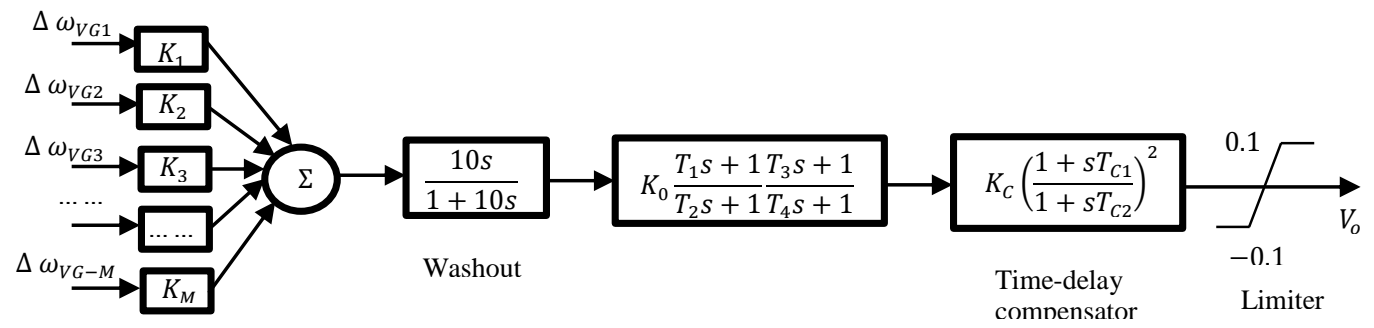

Fig. 3 The proposed structure of VG-PSS. 

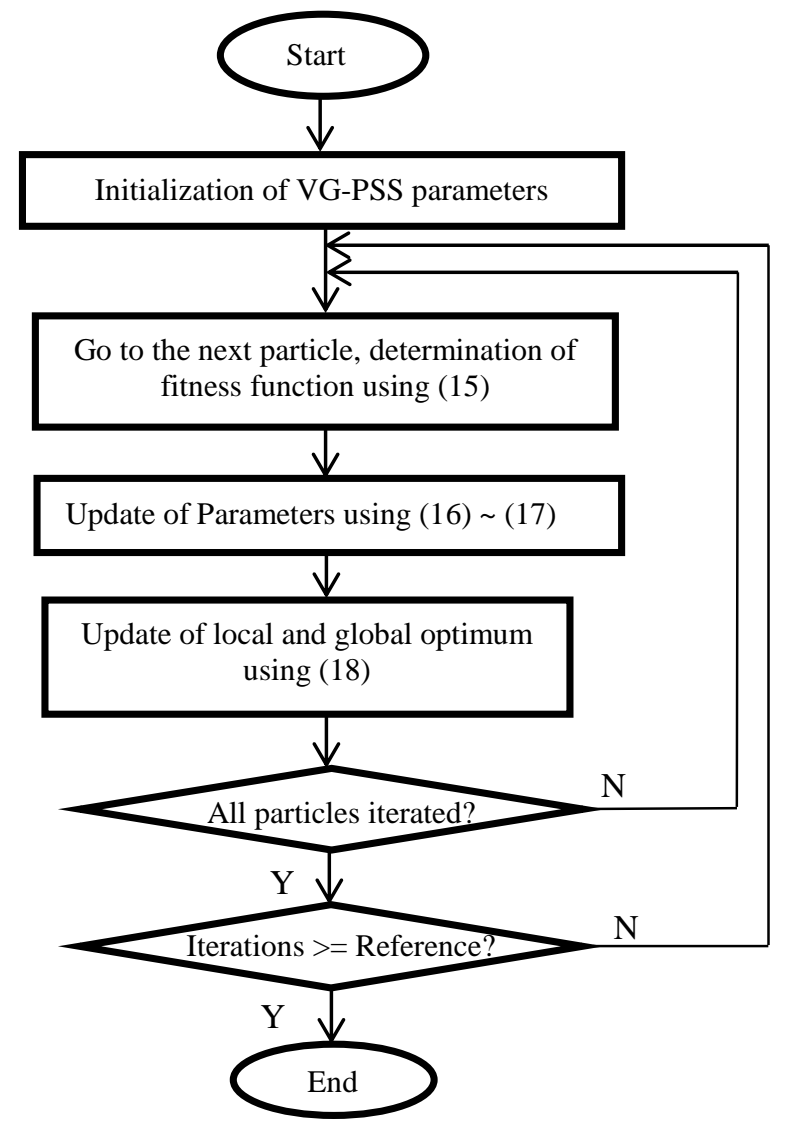

Fig. 4 PSO flowchart for VG-PSS parameters tuning algorithm. 


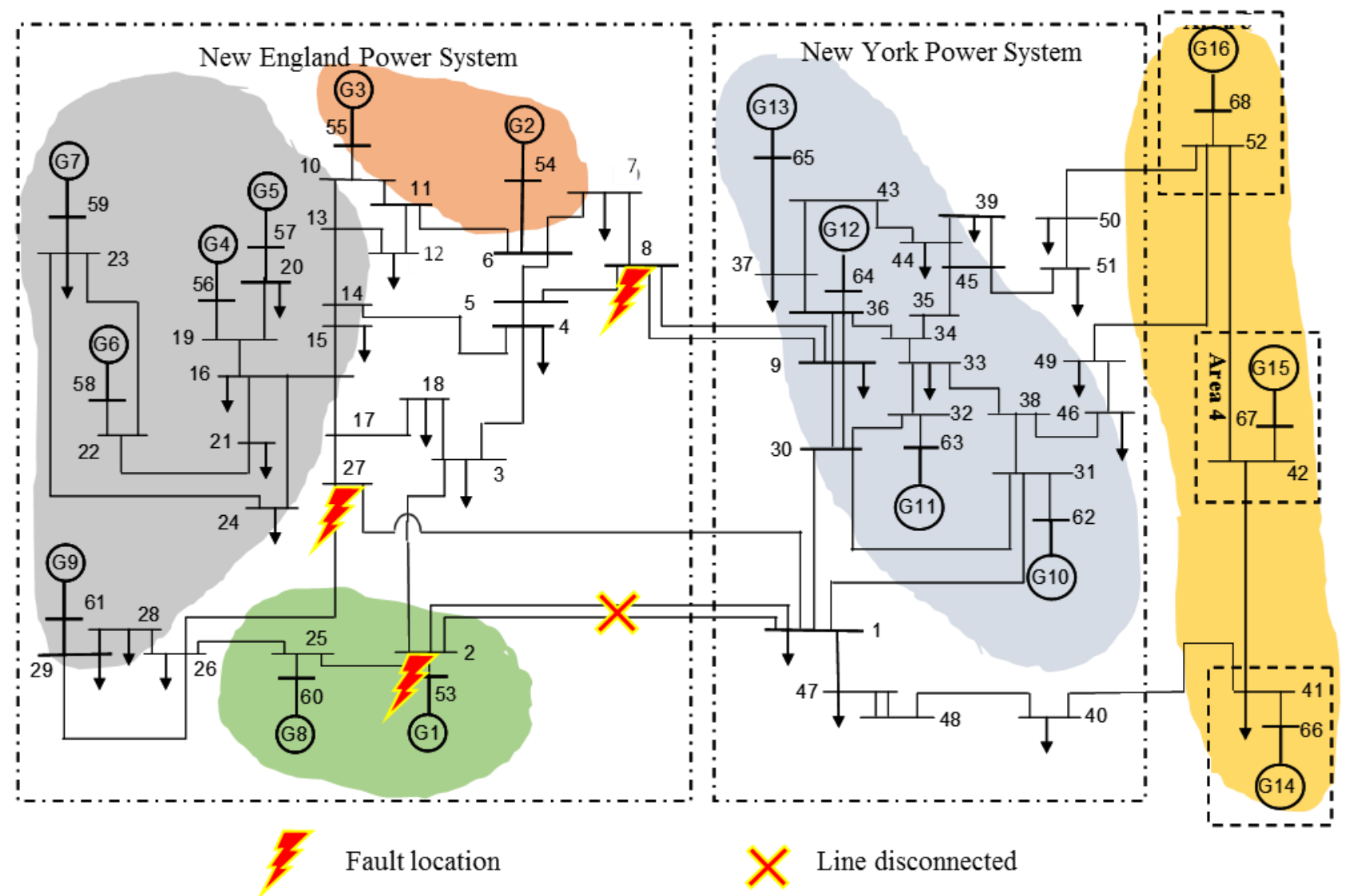

Fig. 5 IEEE 68-Bus 16-machine system. 


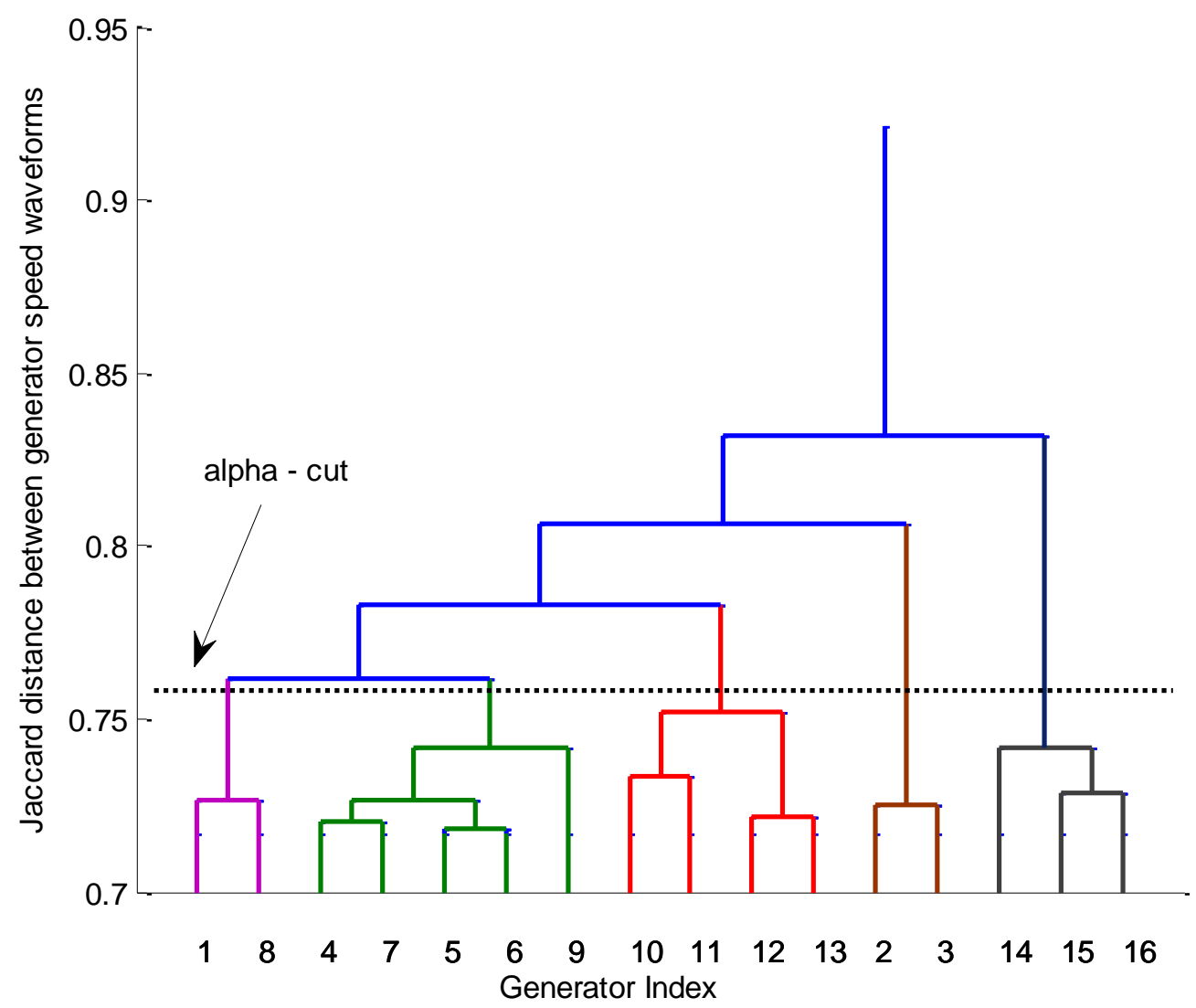

Fig. 6 The dendrogram of clustering result for IEEE 68-Bus system.

The horizontal magenta line shows the clustering threshold 


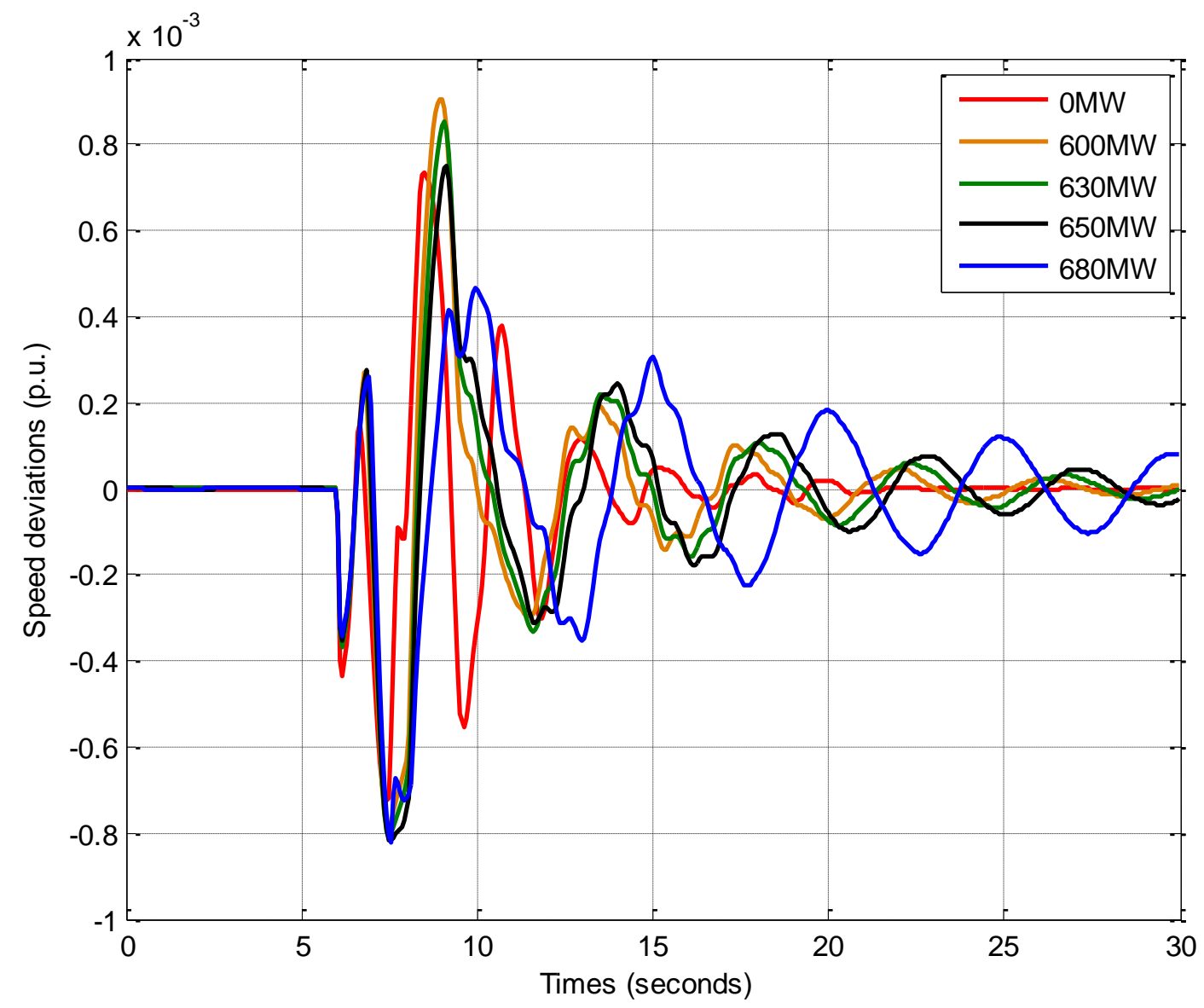

Fig. 7 Speed deviations plots of G15 for different values of $\Delta P$ (increased active power transfer from NE system to NY system) post-fault at Bus 31 . 


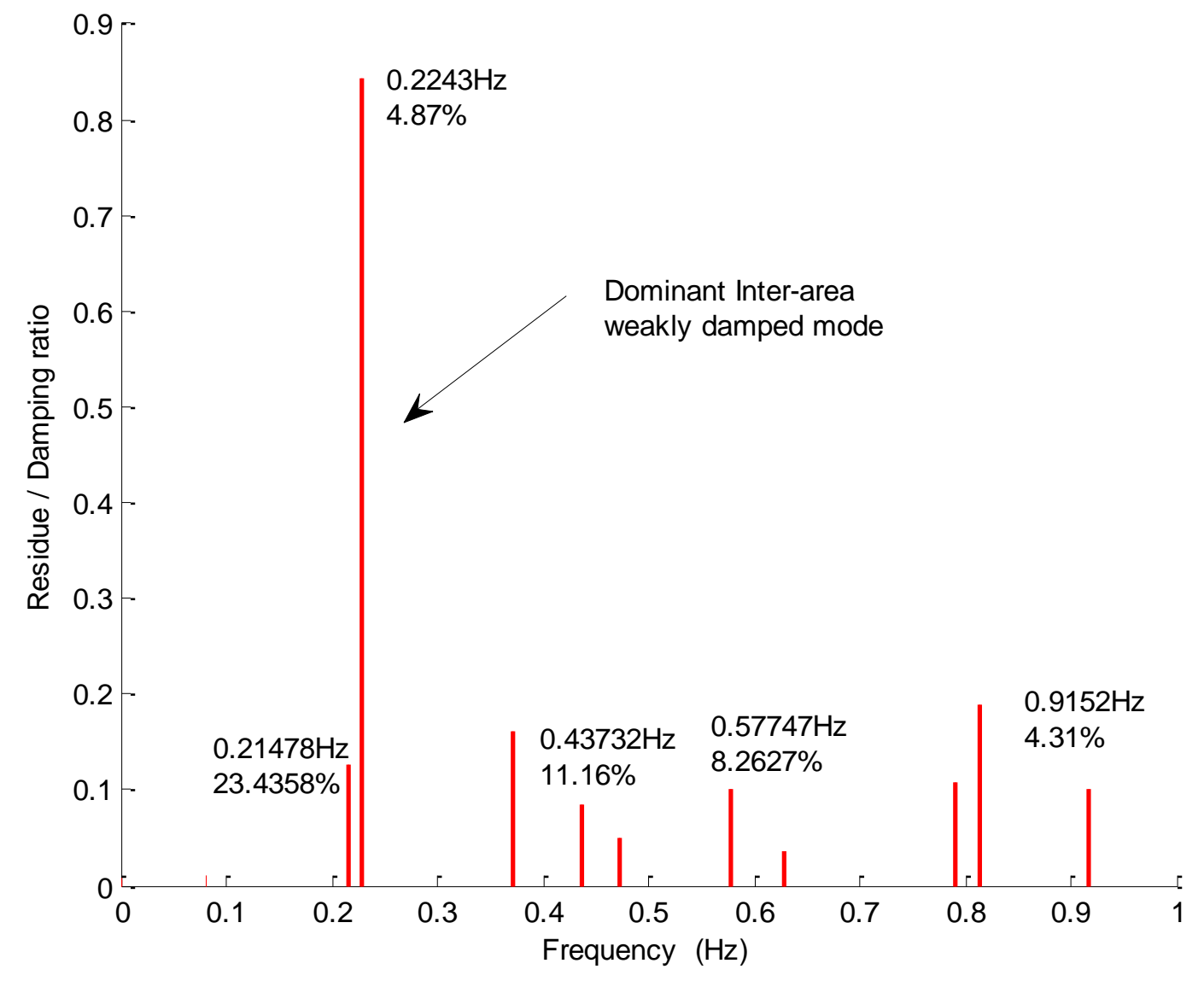

Fig. 8 Identified system modes for $\Delta P=680 \mathrm{MW}$. 


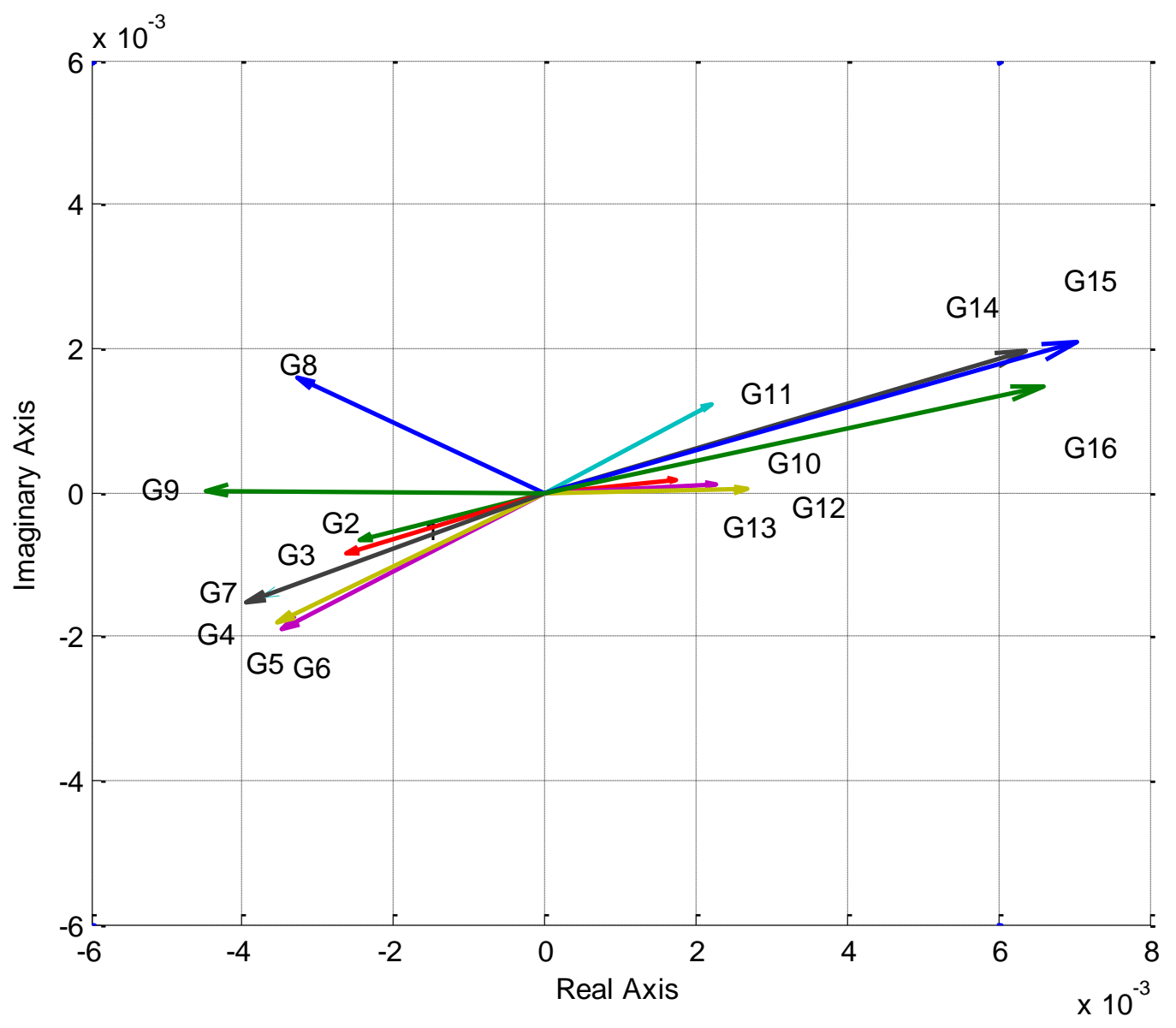

Fig. 9 The phasor plot of observability factors of G1 G16. 


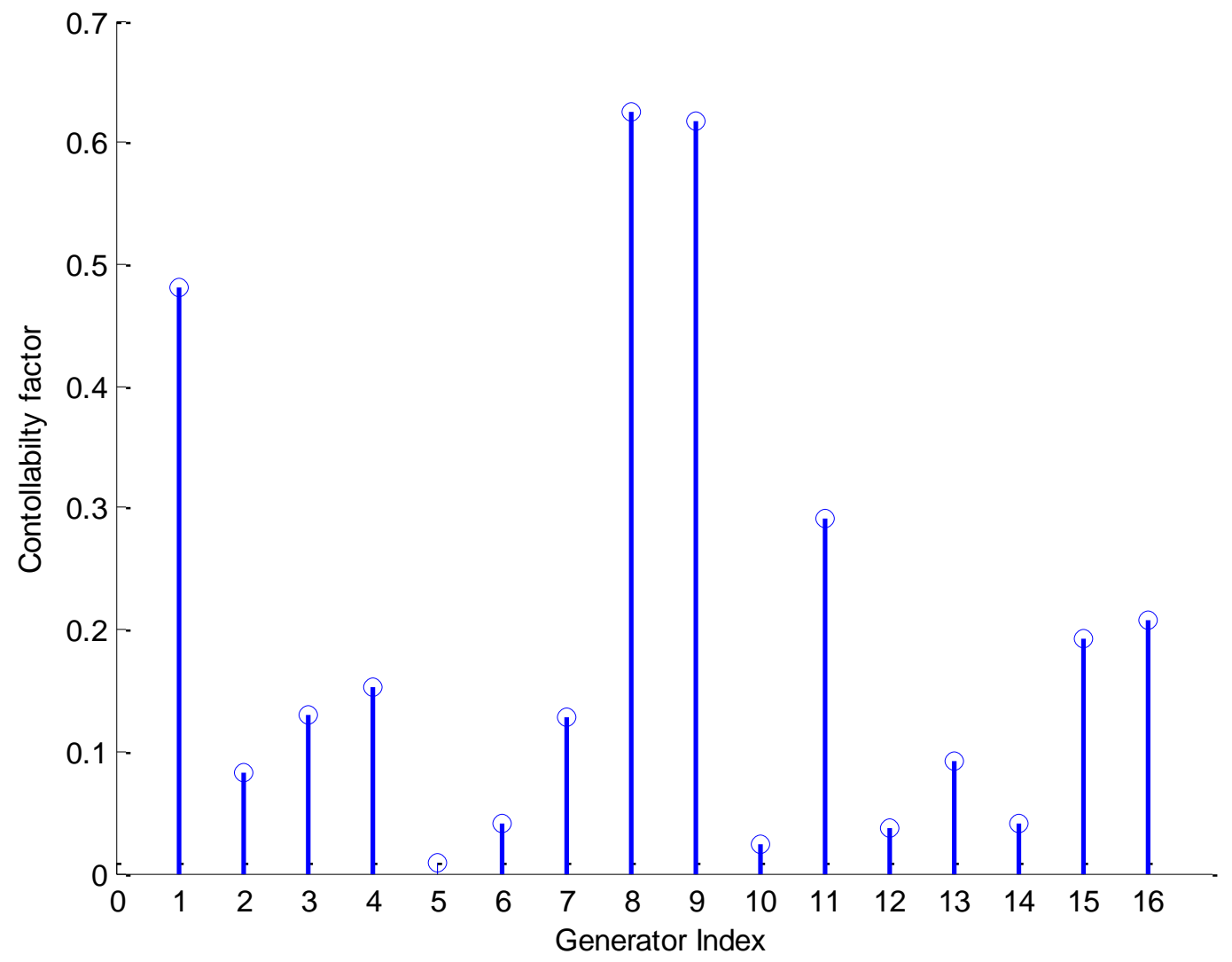

Fig. 10 The controllability factors of G1 G16. 


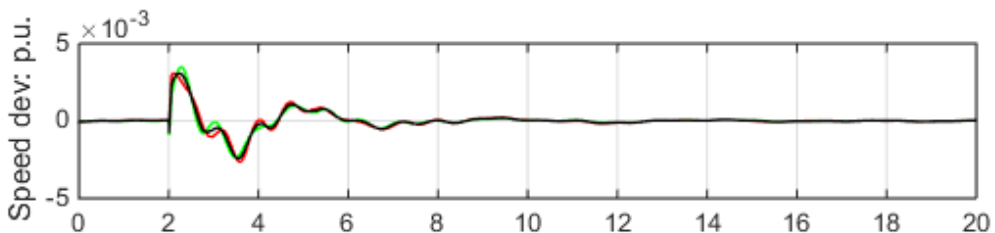

(a)

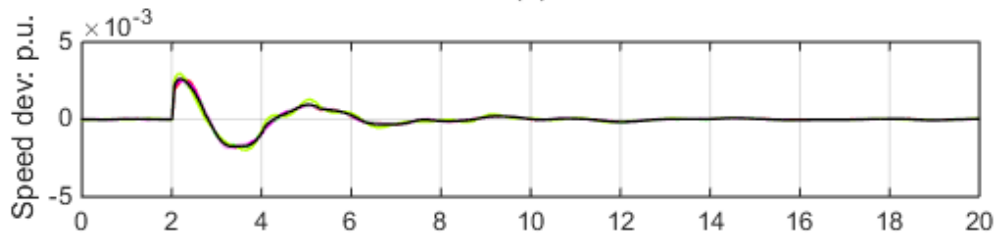

(b)

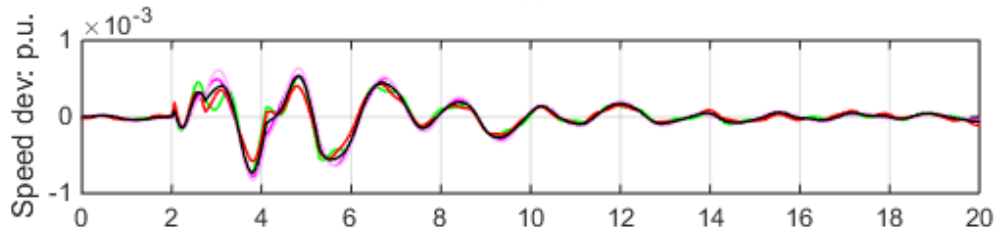

(c)
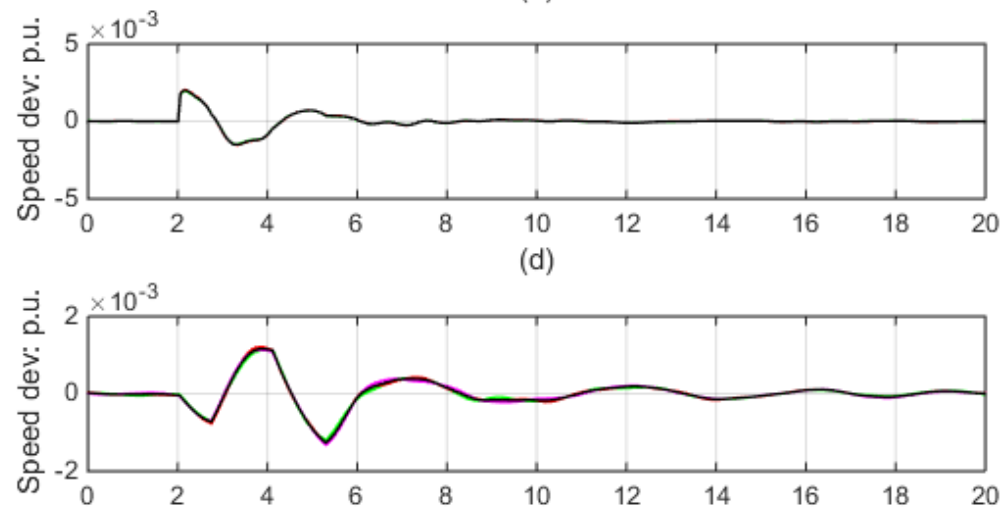

(e)

Time : s

Fig. 11 Speed responses of generators in the five coherent groups.
(a) Group 1: G1,G8
(b) Group 2: G2,G3

(c) Group 3: G4,G5,G6,G7,G9

(d) Group 4: G10,G11,G12,G13

(e) Group 5: G14,G15,G16

Marked in solid black are the virtual generator speed deviations 

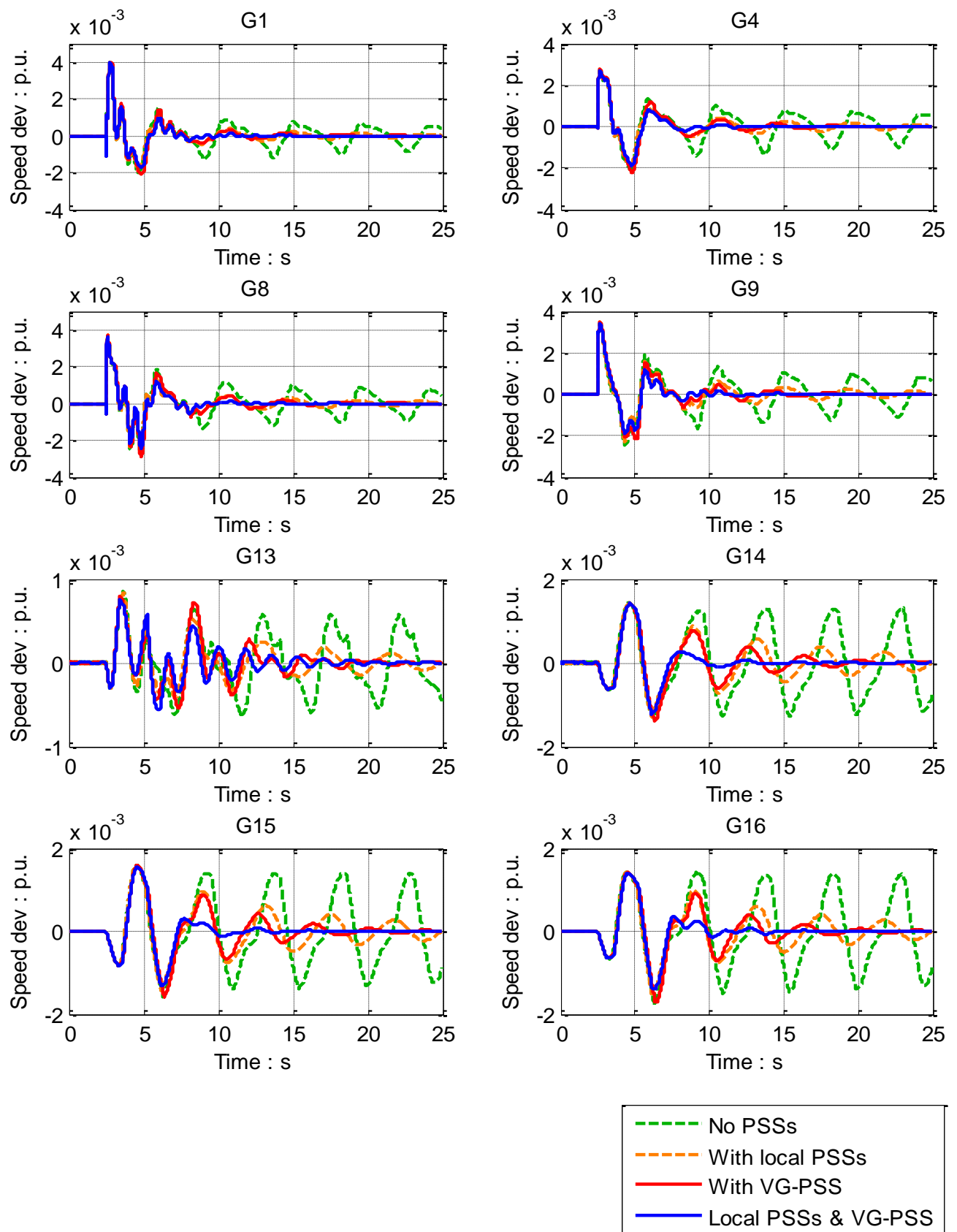

Fig. 12 Case Study I -- The speed deviations plots of selected generators with VG-PSS on G9. 

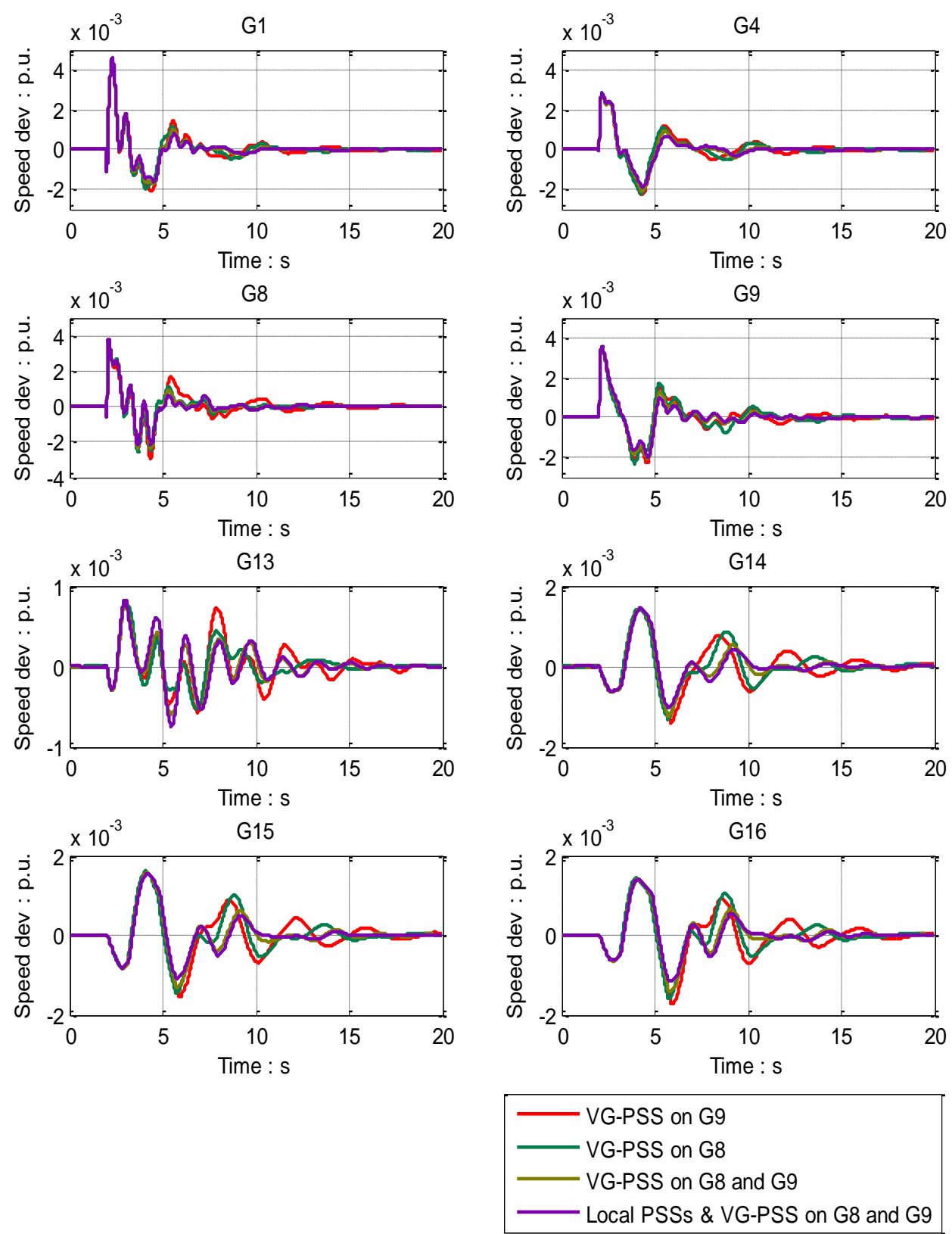

Fig. 13 Case Study I -- The speed deviations plots of selected generators for different sites of VG-PSS installations. 

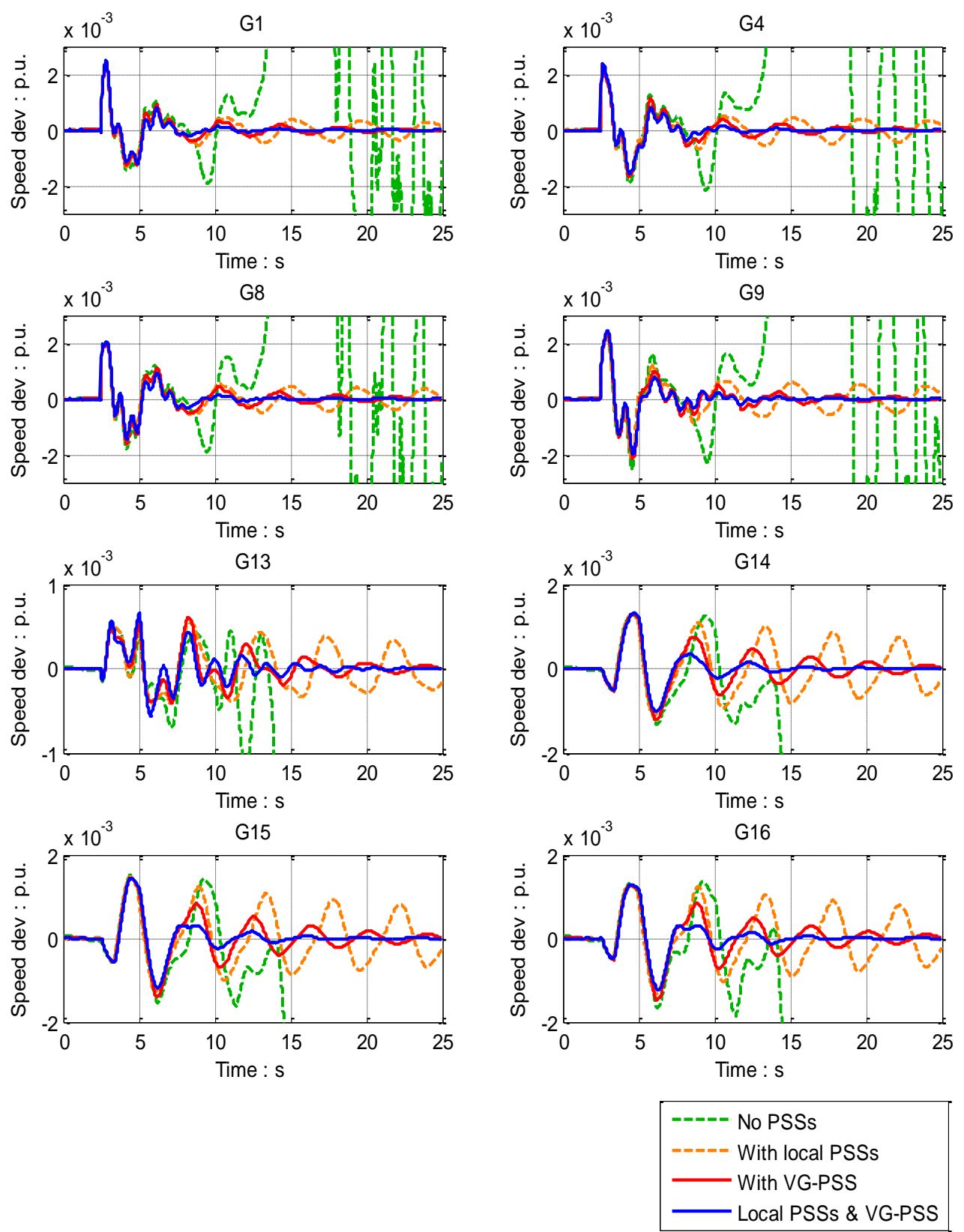

Fig. 14 Case Study II.A -- The speed deviations plots of selected generators with VG-PSS on G9. 

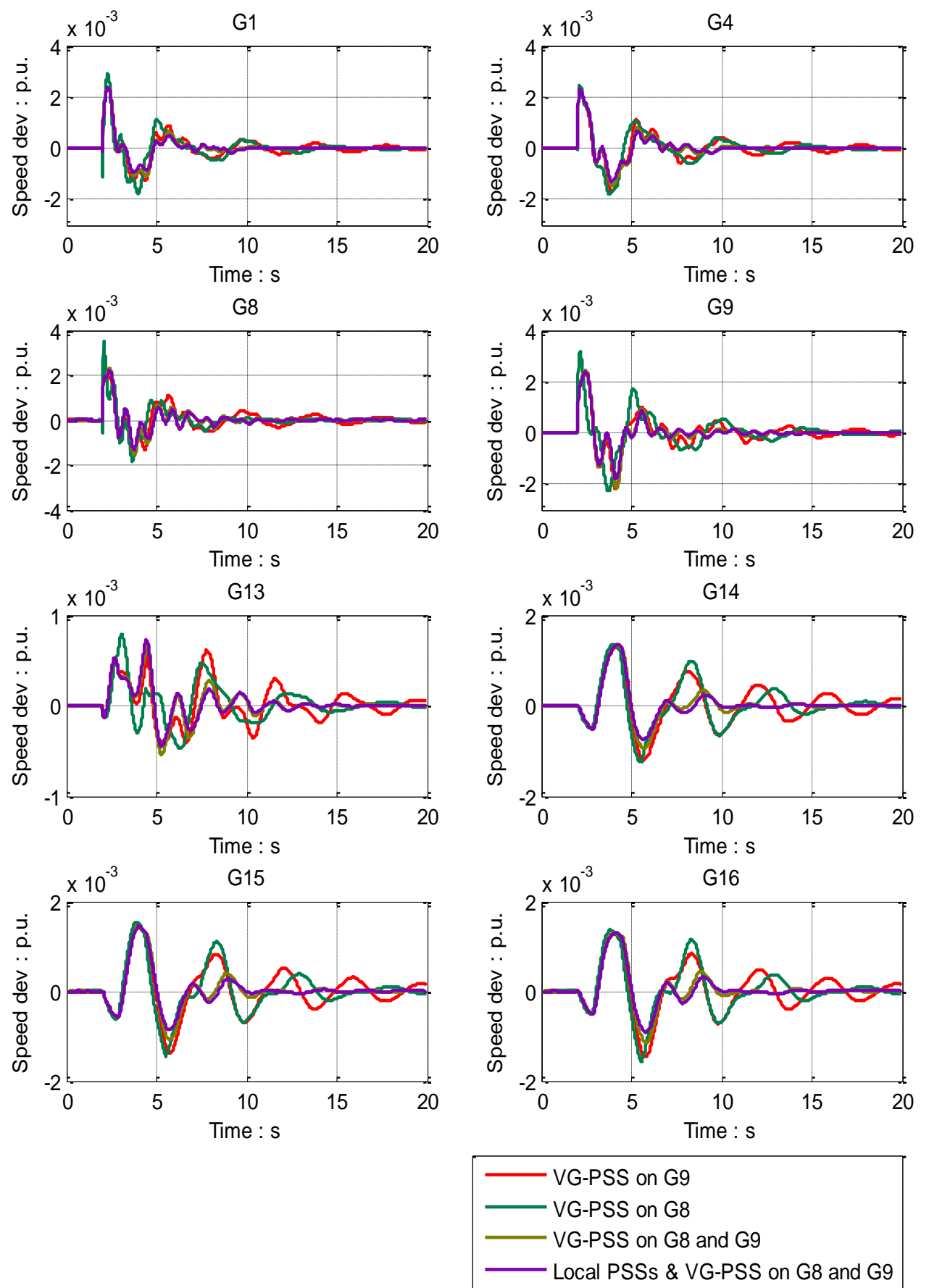

Fig. 15 Case Study II.A -- The speed deviations plots of selected generators for different sites of VG-PSS installations. 

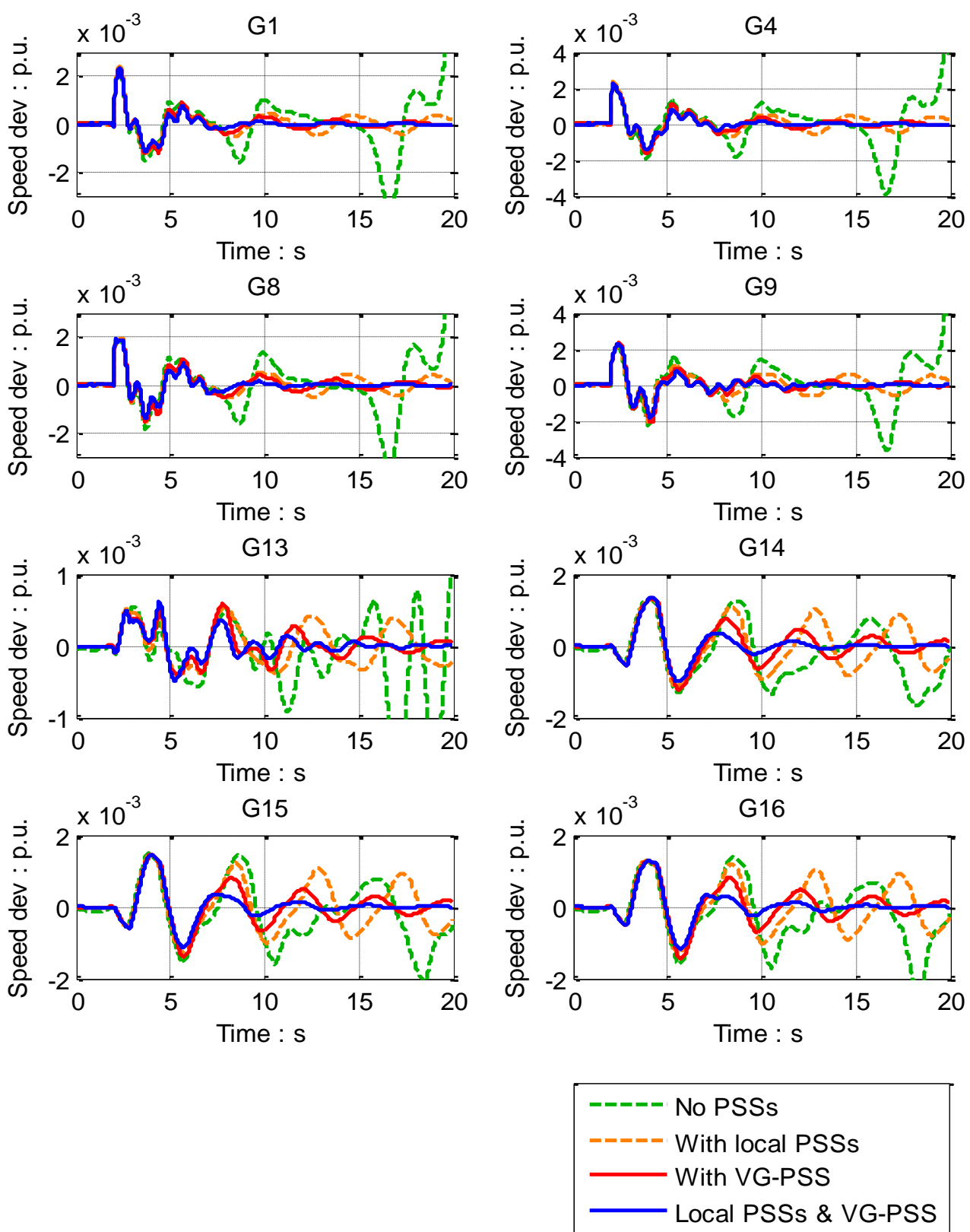

Fig. 16 Case Study II.B -- The speed deviations plots of selected generators with VG-PSS on G9 under a new load condition 

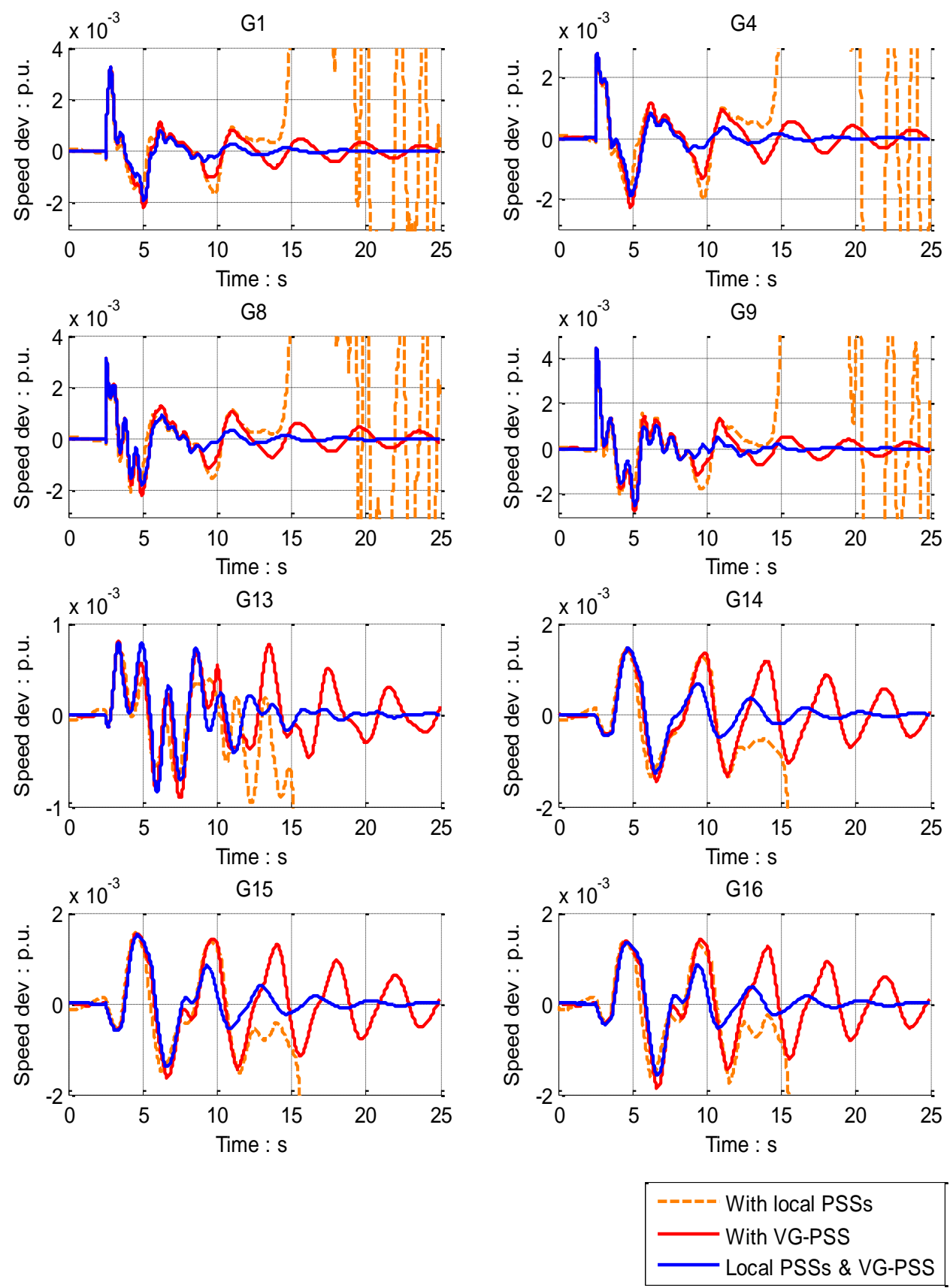

Fig. 17 Case Study III -- The speed deviations plots of selected generators with VG-PSS on G9. 

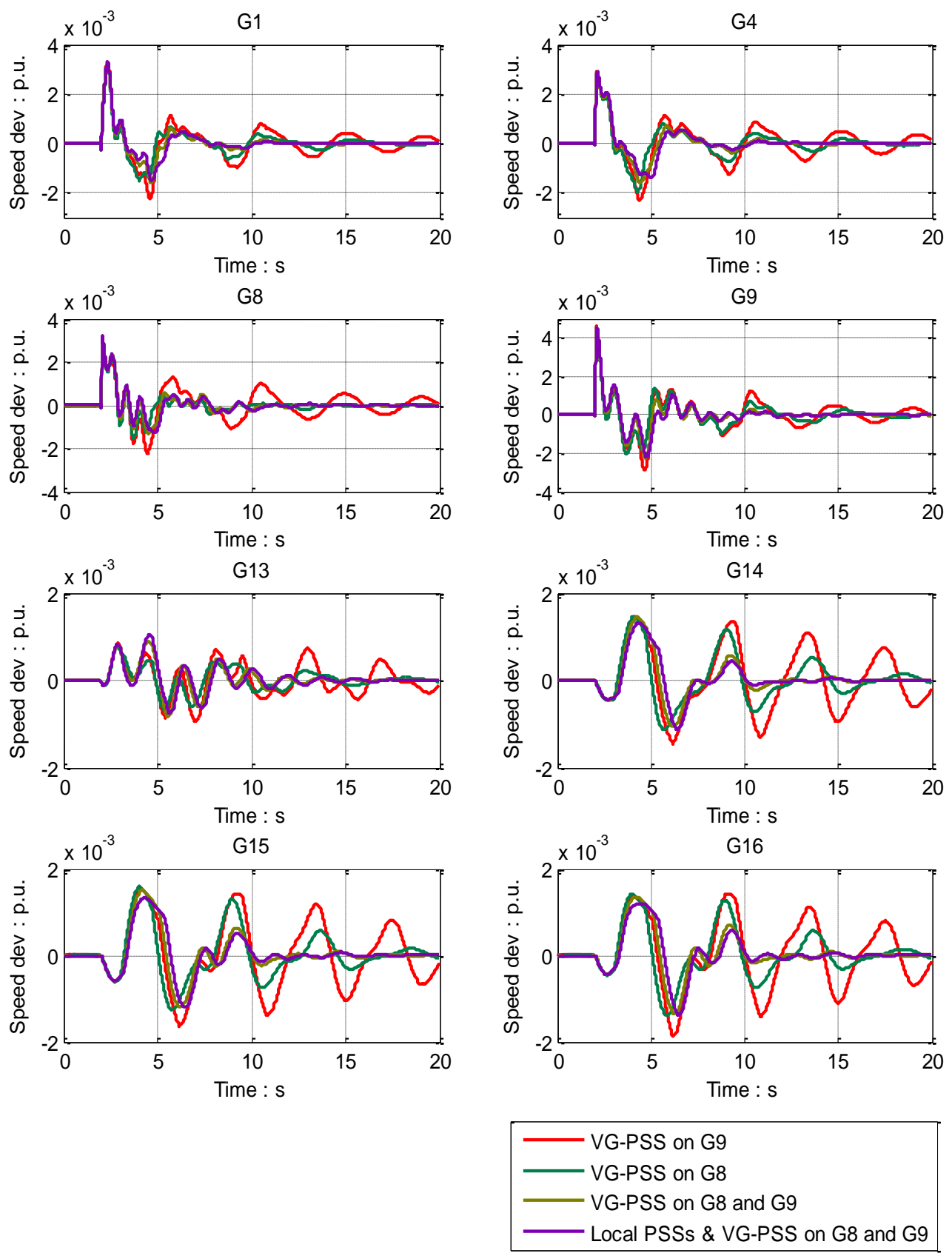

Fig. 18 Case Study III -- The speed deviations plots of selected generators for different sites of VG-PSS installations. 


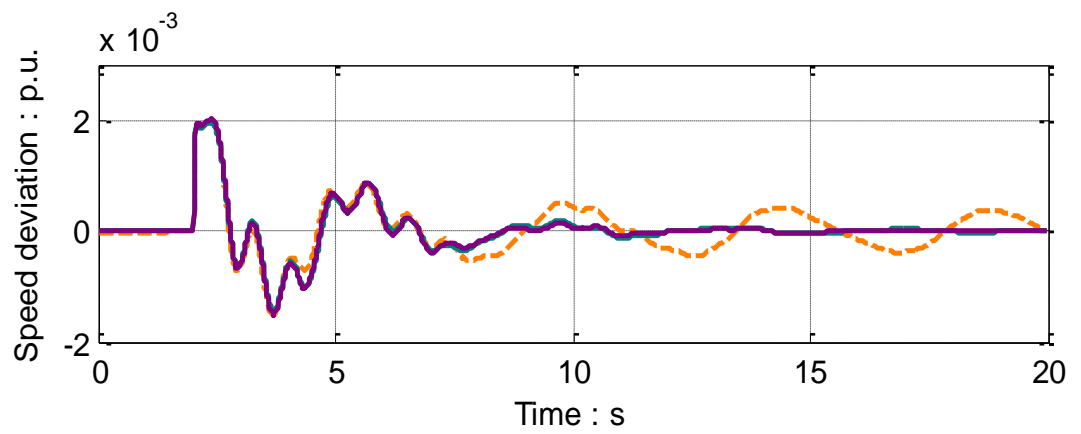

(a)

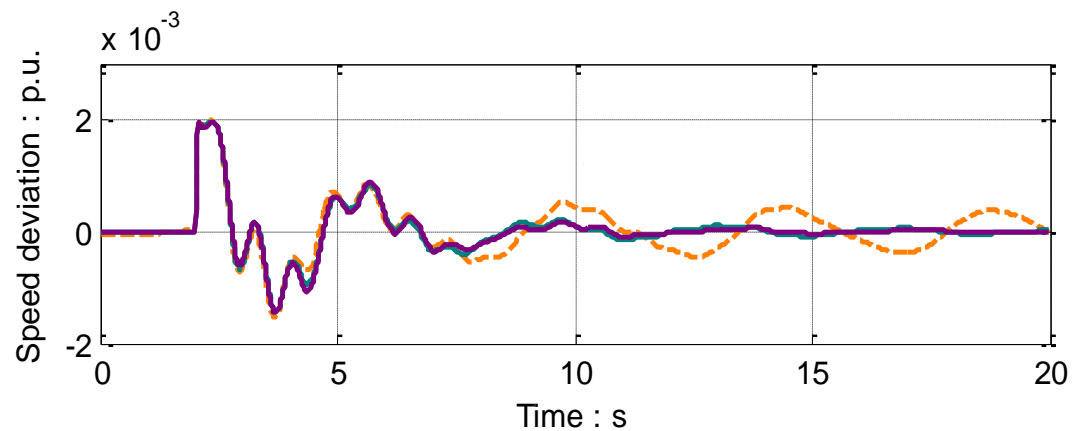

(b)

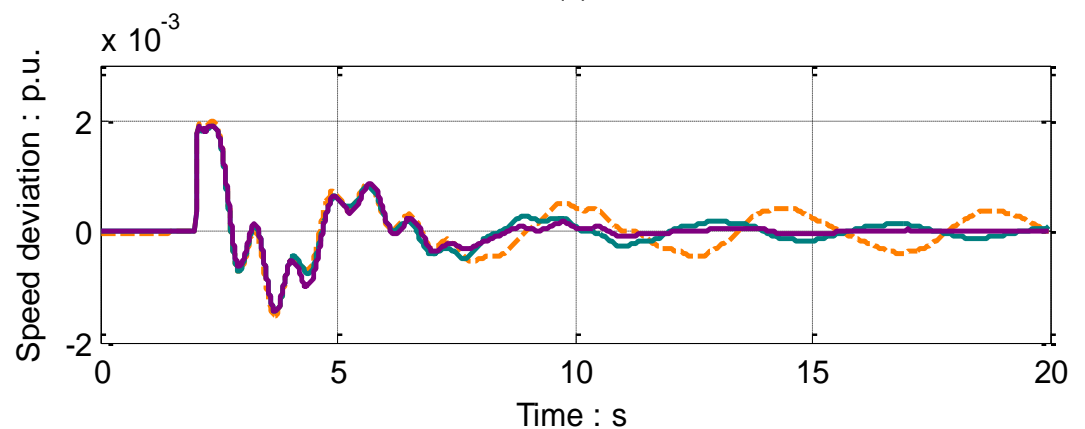

(c)

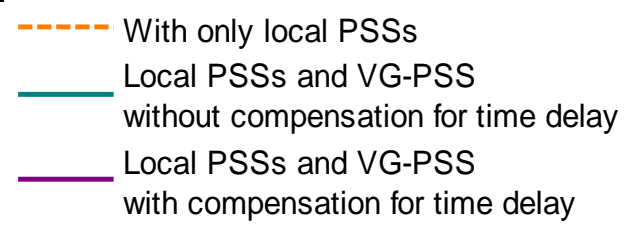

Fig. 19 Impact of time delay for the speed responses at G9.
(a) 50ms time delay
(b) $100 \mathrm{~ms}$ time delay
(c) $200 \mathrm{~ms}$ time delay 
Table I Oscillation frequencies and Damping ratios under multiple cases.

\begin{tabular}{c|c}
\hline$\Delta P(\mathrm{MW})$ & $\begin{array}{c}\text { Mode frequency and corresponding } \\
\text { damping ratio }\end{array}$ \\
\hline 600 & $0.2235 \mathrm{~Hz}, 11.53 \%$ \\
\hline 630 & $0.225 \mathrm{~Hz}, 9.16 \%$ \\
\hline 650 & $0.2233 \mathrm{~Hz}, 7.63 \%$ \\
\hline 680 & $0.2173 \mathrm{~Hz}, 4.74 \%$ \\
\hline 700 & $0.2239 \mathrm{~Hz}, 2.04 \%$ \\
\hline 720 & $0.2112 \mathrm{~Hz}, \quad 1.05 \%$ \\
\hline 740 & $0.2034 \mathrm{~Hz},-0.70 \%$ \\
\hline
\end{tabular}

Table II The similarity index between each generator and its nearest virtual generator

\begin{tabular}{c|c|c|c}
\hline Generator & Similarity Index & Generator & Similarity Index \\
\hline G1 (Group1) & $84.6 \%$ & G9 (Group3) & $94.5 \%$ \\
\hline G2 (Group2) & $86.4 \%$ & G10 (Group4) & $74.5 \%$ \\
\hline G3 (Group2) & $86.3 \%$ & G11 (Group4) & $75.8 \%$ \\
\hline G4 (Group3) & $84.6 \%$ & G12 (Group4) & $83.6 \%$ \\
\hline G5 (Group3) & $94.5 \%$ & G13 (Group4) & $84.7 \%$ \\
\hline G6 (Group3) & $92.4 \%$ & G14 (Group5) & $93.1 \%$ \\
\hline G7 (Group3) & $93.6 \%$ & G15 (Group5) & $95.6 \%$ \\
\hline G8 (Group1) & $84.5 \%$ & G16 (Group5) & $93.7 \%$ \\
\hline
\end{tabular}


Table III The optimized parameters of the VG-PSS

\begin{tabular}{c|c|c}
\hline Parameters & $\begin{array}{c}\text { Case I } \\
\text { With VG-PSS on G8 }\end{array}$ & $\begin{array}{c}\text { Case II } \\
\text { With VG-PSS on G9 }\end{array}$ \\
\hline$K_{1}$ & 0.9587 & -0.2318 \\
\hline$K_{2}$ & 0.363 & -0.0524 \\
\hline$K_{3}$ & -1.00 & 0.8703 \\
\hline$K_{4}$ & -0.7126 & 0.5533 \\
\hline$K_{5}$ & 1.00 & 0.9963 \\
\hline$K_{0}$ & 25 & 19.2026 \\
\hline$T_{1}$ & 1.5416 & 0.4680 \\
\hline$T_{2}$ & 0.4024 & 0.7744 \\
\hline$T_{3}$ & 5.2393 & 6.8278 \\
\hline$T_{4}$ & 4.1175 & 2.7501 \\
\hline
\end{tabular}


Table IV Settling time of generator speed deviations for the three case studies (in seconds)

\begin{tabular}{c|c|c|c|c|c}
\hline Cases & $\begin{array}{c}\text { With no PSSs } \\
(\mathrm{s})\end{array}$ & $\begin{array}{c}\text { With only local } \\
\text { PSSs } \\
(\mathrm{s})\end{array}$ & $\begin{array}{c}\text { With only VG-PSSs } \\
\text { on G8 } \\
(\mathrm{s})\end{array}$ & $\begin{array}{c}\text { With only VG-PSSs } \\
\text { on G9 } \\
(\mathrm{s})\end{array}$ & $\begin{array}{c}\text { With both VG-PSSs } \\
\text { and local PSSs } \\
(\mathrm{s})\end{array}$ \\
\hline Case Study I & $>50$ & 28.2 & 14.6 & 16.4 & 6.1 \\
\hline Case Study II.A & Unstable & $>50$ & 26.6 & 27.6 & 7.2 \\
\hline Case Study II.B & Unstable & $>50$ & 24.6 & 26.5 & 7.0 \\
\hline Case Study III & Unstable & Unstable & 36.2 & 40.8 & 8.8 \\
\hline
\end{tabular}




\section{Appendix}

Table A.I The reference of active power generation for all generators

\begin{tabular}{|c|c|c|c|c|c|}
\hline Generator & $\begin{array}{c}\text { Active Power } \\
\text { Generation for } \\
\text { Base Condition } \\
\text { (MW) }\end{array}$ & $\begin{array}{l}\text { Active Power } \\
\text { Generation for } \\
\text { Operating } \\
\text { Condition II } \\
\text { (MW) }\end{array}$ & $\begin{array}{c}\text { Active Power } \\
\text { Generation for } \\
\text { Operating } \\
\text { Condition III.A } \\
\text { (MW) }\end{array}$ & $\begin{array}{c}\text { Active Power } \\
\text { Generation for } \\
\text { Operating } \\
\text { Condition III.B } \\
\text { (MW) }\end{array}$ & $\begin{array}{c}\text { Active Power } \\
\text { Generation for } \\
\text { Operating } \\
\text { Condition IV(MW) }\end{array}$ \\
\hline G1 & 250 & 272.2 & 276.7 & 276.7 & 279.4 \\
\hline G2 & 545 & 567.2 & 571.7 & 571.7 & 574.4 \\
\hline G3 & 650 & 672.2 & 676.7 & 676.7 & 679.4 \\
\hline G4 & 632 & 654.2 & 658.7 & 658.7 & 661.4 \\
\hline G5 & 505.2 & 527.4 & 531.9 & 531.9 & 534.6 \\
\hline G6 & 700 & 722.2 & 726.7 & 726.7 & 729.4 \\
\hline G7 & 560 & 582.2 & 586.7 & 586.7 & 589.4 \\
\hline G8 & 540 & 562.2 & 566.7 & 566.7 & 569.4 \\
\hline G9 & 800 & 822.2 & 826.7 & 826.7 & 829.4 \\
\hline G10 & 500 & 500 & 500 & 500 & 500 \\
\hline G11 & 1150 & 1150 & 1150 & 1150 & 1150 \\
\hline G12 & 1350 & 1350 & 1350 & 1350 & 1350 \\
\hline G13 & 3445 & 3245 & 3250 & 3701.9 & 3180 \\
\hline G14 & 1785 & 1785 & 1785 & 1785 & 1785 \\
\hline G15 & 1000 & 1000 & 1000 & 1000 & 1000 \\
\hline G16 & 4000 & 4000 & 4000 & 4000 & 4000 \\
\hline
\end{tabular}


References

[1] S. Ray, G. K. Venayagamoorthy, "Wide-area signal-based optimal neuro-controller for a UPFC", IEEE Transactions on Power Delivery, pp. 1597-1605, vol. 23, 2008.

[2] D. J. Trudaowski, J. R. Smith, T.A. Short, D. A. Pierre, "An application of prony methods in PSS design for multimachine systems", IEEE Transactions on Power Systems, vol. 6, pp. 118-126, 1991.

[3] P. Palangpour, P. Mitra, G. K. Venayagamoorthy, "DSP-based PSO implementation for online optimization of power system stabilizers", AHS '08 NASA/ESA Conference on Adaptive Hardware and Systems, pp. 379-384, 2008.

[4] K. T. Law, D. J. Hill, N. R. Godfrey, "Robust co-ordinated AVR-PSS design", IEEE Transactions on Power Systems, vol. 9, pp. 1218-1225, 1994.

[5] A. Dysko, W. E. Leithead, J. O'Reilly, "Enhanced power system stability by coordinated PSS design", IEEE Transactions on Power Systems, vol. 25, pp. 413-422, 2010.

[6] A. Hasanovic, A. Feliachi, A. Hasanovic, N. B. Bhatt, A. G. DeGroff, "Practical robust PSS design through identification of low-order transfer functions", IEEE Transactions on Power Systems, vol. 19, pp. 1492-1500, 2004.

[7] C. W. Taylor, Deninis C. Erickson, K. E. Martin, R. E. Wilson, V. Venkatasubramanian, "WACS-wide-area stability and voltage control system: R\&D and online demonstration", Proceedings of the IEEE, vol. 93, pp. 892-906, 2005.

[8] S. Ray, G. K. Venayagamoorthy, "Real-time implementation of a measurement-based adaptive wide-area control system considering communication delays", IET Generation, Transmission \& Distribution, Vol. 2, pp. 62-70, 2008.

[9] A. Heniche, I. Kamwa, "Assessment of two methods to select wide-area signals for power system damping control”, IEEE Transactions on Power Systems, vol. 23, pp. 572-581, 2008.

[10] P. Mitra, G. K. Venayagamoorthy, "Wide area control for improving stability of a power system with plug-in electric vehicles", IET Generation, Transmission \& Distribution, vol. 4, pp. 1151-1163, 2010.

[11] M. Chilali and P. Gahinet, " $\mathrm{H}_{\infty}$ design with pole placement constraints: An LMI approach," IEEE Trans. Automation and Control, vol. 41, pp. 358-367, 1996.

[12] D. Molina, G. K. Venayagamoorthy, Jiaqi Liang, R. G. Harley, "Intelligent local area signals based damping of power system oscillations using virtual generators and approximate dynamic programming", IEEE Transactions on Smart Grid, vol. 4, pp. 498-508, 2013.

[13] X. M. Mao, Y. Zhang, L. Guan, X. C. Xu, et al, "Improving power system dynamic performance using widearea high-voltage direct current damping control", IET Generation, Transmission \& Distribution, vol. 2, pp. 245-251, 2008.

[14] B. Yang, Y. Sun, "Damping factor based delay margin for wide area signals in power system damping control", IEEE Transactions on Power Systems, vol. 28, pp. 3501-3502, 2013.

[15] C. N. Taranto, D. M. Falcao, "Robust decentralised control design using genetic algorithms in power system damping control", IEE Proceedings Generation, Transmission and Distribution, vol. 145, pp. 1-6, 1998

[16] R. Majumder, B. Chaudhuri, B. C. Pal, Q. Zhong, "A unified Smith predictor approach for power system damping control design using remote signals", IEEE Transactions on Control System Technology, vol. 13, pp. 1063-1068, 2005.

[17] V. G. D. C. Samarasinghe, N. C. Pahalawaththa, "Damping of multimodal oscillations in power systems using variable structure control techniques", IEE Proceedings Generation Transmission and Distribution, vol. 144, pp. 323-331, 1997

[18] N. J. Bershad, J. C. M. Bermudez, J. Y. Tourneret, "Stochastic analysis of the LMS algorithm for system identification with subspace inputs", IEEE Transactions on Signal Processing, pp. 1018-1027, vol. 56, 2008.

[19] Paul H. Bardell, William H. McAnney, Jacob Savir, "Built-In Test for VLSI: Pseudorandom Techniques", John Wiley \& Sons, New York, 1987.

[20] K. Tang, G. K. Venayagamoorthy, "Online coherency analysis of synchronous generators in a power system", 2014 IEEE PES Innovative Smart Grid Technologies Conference, 2014.

[21] Y. del Valle, G. K. Venayagamoorthy, S. Mohagheghi, "Particle Swarm Optimization: Basic Concept, Variants and Applications in Power Systems", IEEE Transactions on Evolutionary Computation, vol. 2, pp. 171-195, 2008. 
[22] B. C. Pal, "Robust pole placement versus root-locus approach in the context of damping interarea oscillations in power systems", IEE Proceedings Generation, Transmission and Distribution, vol. 149, pp. 739-745, 2002.

[23] S. Ray, G. K. Venayagamoorthy, B. Chaudhuri, R. Majumder, "Comparison of adaptive critic-based and classical wide-area controllers for power systems", IEEE Systems, Man, and Cybernetics, Part B: Cybernetics, pp. 1002-1007, vol. 38, 2008

[24] T. K. Das, G. K. Venayagamoorthy, U. O. Aliyu, "Bio-inspired algorithms for the design of multiple optimal power system stabilizers: SPPSO and BFA", IEEE Transactions on Industry Application, pp. 1445-1457, vol. 44. 2008.

[25] P. Zhang, D. Y. Yang, K. W. Chan, G. W. Cai, "Adaptive wide-area damping control scheme with stochastic subspace identificiation and signal time delay compensation", IET Generation, Transmission \& Distribution, pp. 844-852, vol. 6, 2012.

[26] K. Tang, G. K. Venayagamoorthy, “An LMI-SSI model based PSS design approach for a multi-machine power system”, 2015 Clemson University Power System Conference, pp. 1-7, 2015. 